\title{
Improving Performance and Quantifying Uncertainty of Body- Rocking Detection using Bayesian Neural Networks
}

This paper was downloaded from TechRxiv (https://www.techrxiv.org).

\section{LICENSE}

CC BY 4.0

SUBMISSION DATE / POSTED DATE

10-10-2021 / 14-10-2021

\section{CITATION}

da Silva, Rafael Luiz; Zhong, Boxuan; Chen, Yuhan; Lobaton, Edgar (2021): Improving Performance and Quantifying Uncertainty of Body-Rocking Detection using Bayesian Neural Networks. TechRxiv. Preprint. https://doi.org/10.36227/techrxiv.16779301.v1

$\mathrm{DOI}$

10.36227/techrxiv.16779301.v1 


\title{
Improving Performance and Quantifying Uncertainty of Body-Rocking Detection using Bayesian Neural Networks
}

\author{
Rafael L. da Silva, Boxuan Zhong, Yuhan Chen and Edgar Lobaton
}

\begin{abstract}
Body-rocking is an often-undesired stereotypical motor movement performed by some individuals, and its detection is essential for self-re-education and habit change. We envision a pipeline that includes inertial wearable sensors streaming data to a smart aggregator that performs the detections in real-time. Notifications can then be sent to the user to change such behavior. For this task, having precise detection is essential to keep the user engaged and without the discomfort of too many false detections, leading to alarm fatigue. We present a pipeline using Bayesian Neural Networks for uncertainty quantification in the model and the data, as an aid to improve and identify ambiguity in the predictions, aiming to obtain accurate body-rocking detections. This allows us to improve performance and provide a better trade-off between precision and recall. A comprehensive analysis comparing several models is performed to evaluate the impact of model capacity on the Bayesian approach. We show that higher model capacity alone is not able to extract a higher performance, while pairing it with the Bayesian approach does yield significant improvements. Disparities in uncertainty quantification are more accurately quantified by calibrating them using deep neural networks. We show that the calibrated probabilities are effective quality indicators of reliable predictions. Finally, we show that the selection of reliable detections using uncertainty allows an enhanced response suppressing false positives.
\end{abstract}

\section{Index Terms}

Bayesian neural networks, uncertainty quantification, stereotypical motor movement, body-rocking

\section{INTRODUCTION}

Body-rocking is one type of Stereotypical Motor Movements (SMM) observed in normal children (in medical literature referred to as "primary" cases) and in children presenting symptoms of distinct mental disorders ("secondary" cases). Such movements are normally involuntary and recurrent, sometimes nonrhythmic and purposeless. For secondary cases, SMM has partial overlap with developing disorders such as autism spectrum disorder (ASD), obsessive compulsive disorder (OCD), obsessive compulsive behavior (OCB), as well as self-destructive behavior (SDB), Tourette syndrome (TS) and attention deficit hyperactivity disorder (ADHD), [1], [2]. Such repetitive behavior could be, but is not limited to, for instance hand flapping, body-rocking or a combination of the two. Several possible triggers have been investigated for this behavior and some indications have shown a link between such patterns with excitement and anxiety [3]. The definition and diagnostic of SMM and their respective mental disorder has been argued as vague and imprecise, although the diagnostic and statistical manual of mental disorders (DSM-IV) criteria for stereotypes requires repetitive, non-functioning behaviors for more than 4 weeks and interfering in normal activities or resulting in self injury [4]. Body-rocking has been shown to be common in blind infants [5] and to difficult social relationships [6]. A survey with college undergraduates has shown that self-reported body-rocking is connected to General Anxiety Disorder, [7]. For primary cases of body-rocking, the authors in [8] have shown that habit reversal and differential reinforcement is beneficial for self-awareness and stopping of the movement, which is also summarized in table 1 of [9].
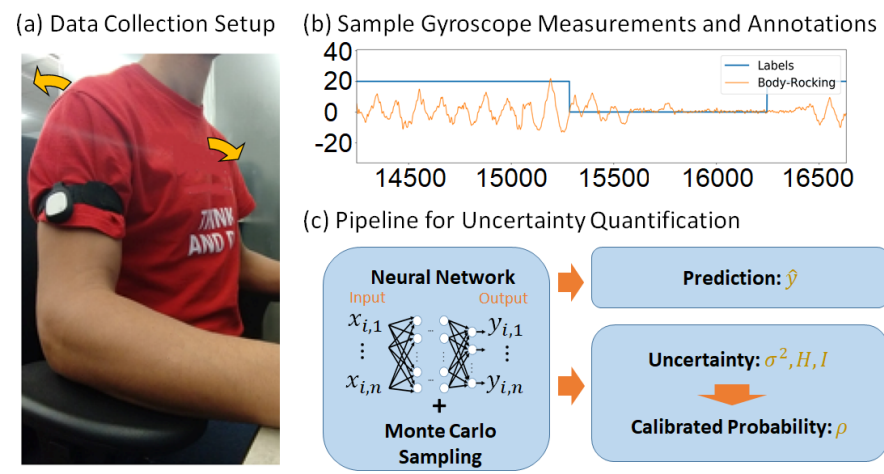

(c) Pipeline for Uncertainty Quantification

Fig. 1: Illustration of the data collection, prediction and uncertainty quantification pipelines. (a) Body-rocking movement illustration with sensor placed on the arm. The arrows indicate the forward and backward body-rocking. (b) A sample gyroscope measurement with corresponding annotations. (c) Our pipeline for prediction and uncertainty which utilizes a Bayesian Neural Network framework based on Monte Carlo (MC) Sampling from dropout. The uncertainty measures include a variance $\sigma^{2}$ indicative of observation noise, and entropy $H$ and mutual information $I$ obtained from the MC samples. This framework improves the performance of prediction and yields a calibrated probability $\rho$ that is reliably indicating our confidence on a prediction.

This work is supported by the National Science Foundation (NSF) under award CNS 1552828. R. L. da Silva, B. Zhong, Y. Chen and E. Lobaton are with the Department of Electrical and Computer Engineering of NC State University, Raleigh, NC, 27695 USA, e-mails: rafaelonwork@gmail.com. 
Since medical research has shown potential for improve-

ments through re-education, multiple efforts have been put together to identify and characterize reliably the occurrence of body-rocking for both cases aforementioned, envisioning early diagnosis and self-awareness for behavior reversal.

Wearable sensors such as inertial measurement units (IMU) equipped with 3 axis accelerometers and gyroscopes have been appropriate for this purpose since they are non invasive, light weight and may be placed on virtually any body limb. However, signals collected among different individuals demonstrate variability in their properties that proposed algorithms for body-rocking detection have been challenged to deal with.

Real-time implementation notification systems have been attempted along with a viability study [10] revealing an upper bound for real time implementation but haven't provided good accuracy or detection results. Fast response and precision are key factors for accurate detection that engages the user to keep wearing the system. From our observations, we noticed that false negatives are more tolerable than false positives to minimize effects similar to "alert fatigue" [11], [12], thus precision is potentially more important than recall. In order to address that, Bayesian neural networks (BNN) [13]-[15] could play an important role due to their capability of uncertainty quantification. Such networks have been shown to be versatile frameworks, which have been used for prosthesis applications [16], [17], control and robotics [18], and active recognition [19] to name a few. Uncertainty quantification could provide bounds to consider or discard a model's prediction based on a desired trade-off between precision and recall.

In this work, we present a framework for detection of body-rocking activity. We take advantage of the uncertainty quantification provided by BNNs to stipulate a confidence for a given prediction. The findings and contributions of this paper can be summarized as follows:

1) Our Bayesian framework provides better performance given that there is enough capacity in the model and the performance is also less sensitive to overfitting,

2) We also show that higher capacity alone is not enough for extracting the highest performance found in a given model, when compared to the Bayesian framework,

3) Our Transfer Learning methodology did not improve performance but it prevented the calibrated probability from degrading as model capacity increases,

4) The calibrated probability obtained from our Bayesian framework provides an interpretable quantity that accurately represents the likelihood of correctness of the prediction of the specific dataset,

5) By using the calibrated probability as a criterion for selecting reliable detection, we observe a clear improvement on precision with relatively low trade-off in other metrics (e.g., F1-score).

With that, we argue that reliable detection would be possible for real-time notification systems. The paper is organized as follows: section III provides a literature overview on detection of rocking motion. Section III-A presents the data collection system. The BNN framework, models used, pre-processing and evaluation strategies are included in sections III-B, III-D and III-E respectively. Finally, the results and discussion are presented in sections IV and $\mathrm{V}$ respectively.

\section{RELATED WORK}

There exist three main methodologies in the literature for the detection of rocking motion using machine learning: (1) using handcrafted features, (2) learning features from the data, or (3) a mix of the two.

The first methodology, handcrafted features, is the most popular. The study in [20] places wearable accelerometers on the chest as well as in front of a t-shirt collar for individuals with ASD presenting body-rocking with the goal of identifying such behavior. A zero crossing method is applied on the time domain signals based on typical interval ranges and signal amplitudes between body-rocking motions where the best performance (84\%) was obtained from the chest sensor. The study reported at [21] made use of recurrence plots which identifies similarities by means of Euclidean distance between accelerometer signals representing similar trajectories in a 3D phase space. The authors show that this methodology is orientation invariant and claim resilience to disturbances caused by differences in amplitudes. Recurrence quantification analysis (RQA) was employed as a feature extraction method where characteristics such as amount of recurrences, determinism and entropy were paired with a random forest (RF) algorithm to obtain classification accuracy of up to $86 \%$ on average. They also conducted an interesting analysis to find the best body location for the sensors. As a result, they have found that the sensor placed on the torso contributed the most, based on the output of the RF algorithm. Additionally, using the signals from the torso provided the highest classification accuracy. This result corroborates the findings of [22]. Another approach relying on handcrafted features is the seminal work for body-rocking detection performed by [23] where Stockwell transform, variance, mean difference between axes, entropy and correlation coefficients between axes were used as features to be classified for body-rocking detection with a support vector machine (SVM). Earlier approaches that are similar to the ones mentioned and based on handcrafted features can also be found on [22], [24]-[26].

The second methodology is present in [27] and it is motivated by learning features from raw data in an end-to-end fashion. This work uses a similar deep learning approach to [28] by applying a convolutional neural network (CNN) to raw signals for feature extraction. This approach is applied to the dataset provided by [23] and also their own dataset with simulated SMM and non-SMM activities. The extracted features were classified by long-short term memory (LSTM) recurrent neural networks 
TABLE I: Datasets

\begin{tabular}{lrrrrrrr} 
& Subjects & Sessions & Total Length & Occurrences & Duration of Behavior & Sensors & Limb \\
\hline ESDB & 1 & 14 & $11.74 \mathrm{hrs}$ & 526 & $7 \mathrm{hrs}(59.7 \%)$ & Acc, Gyro & Right upper arm and wrist \\
\hline EDAQA & 6 & 25 & $10.63 \mathrm{hrs}$ & 792 & $2 \mathrm{hrs} \mathrm{(20.3 \% )}$ & Acc & Right/Left wrists, torso \\
\hline
\end{tabular}

(RNN), in a combination of knowledge transfer and ensemble learning to find the best performing model. In an earlier study [29], the same author developed a simpler method where instead of an LSTM for classification, an SVM was used and ensemble learning was not employed. Another very similar study by the same author tried the same configuration but with an LSTM [30], such approach was motivated by the argument that handcrafted features do not capture signal dependencies well, however, the author in [28] showed that handcrafted features still have value for SMM detection. The third methodology is present in the work by the authors of [28]. It is a framework based on transfer learning with the support of CNNs. The CNNs were trained on time-domain and frequency domain representations of the collected signals, where the frequency domain representations were extracted using the Stockwell transform as initially introduced to represent body-rocking signals in [23]. The transfer learning approach is justified by the authors in [28], [31] due to the fact that data domains that share similar characteristics can enhance the ability of algorithms to learn and perform predictions on unknown data. Therefore, the CNNs are used to learn the time-frequency related information of SMM (body rocking, hand flapping, or simultaneous body, rocking and hand flapping) extracted from the dataset created and utilized by [23] and non-SMM related activities (such as walking, sitting, sitting down, standing and standing up) extracted from the PUC dataset [32]. The parameters learned with such training are later used as the transferred knowledge to an SVM classifier. The authors show this approach outperforming all contemporaneous state of the art methods [21], [23], [27] with accuracy and F1-score values capping on average at $98.29 \%$ and $93.66 \%$ respectively.

\section{METHODS}

\section{A. Datasets}

In this work, the public SMM dataset Electrodermal Activity Automated Quality Assessment (EDAQA) $\sqrt{1}$ is used. This dataset was made available initially by [23], and the preprocessing and models by [28] are used as a benchmark. This dataset is split in two trials, Study 1 and Study 2. The sampling frequency of the IMU sensors is different from one trial to another, Study 1 is sampled at $60 \mathrm{~Hz}$ and Study 2 is sampled in $90 \mathrm{~Hz}$. Although the subjects are the same and the two trials are spaced out by two years. This public dataset will be referred as EDAQA dataset. The general caracteristics of EDAQA dataset are described in Table \ with further details in Table $\Pi$.

TABLE II: EDAQA dataset details

\begin{tabular}{l|l|l|l|l} 
& \multicolumn{2}{c}{ Study 1 } & Study 2 \\
\cline { 2 - 5 } & Activity \% & Length (min) & Activity \% & Length (min) \\
\hline Subject 1 & 32.29 & 50.24 & 28.28 & 56.75 \\
Subject 2 & 0.63 & 32.05 & 22 & 51.09 \\
Subject 3 & 5.79 & 64.46 & 0.0425 & 75.92 \\
Subject 4 & 37.035 & 38.867 & 15.081 & 87.473 \\
Subject 5 & 13.905 & 44.68 & 22.567 & 55.18 \\
Subject 6 & 88.5905 & 55.755 & 82.43 & 25.263 \\
\hline Average & $\mathbf{2 9 . 7 1}$ & $\mathbf{4 7 . 6 8}$ & $\mathbf{2 8 . 4 0}$ & $\mathbf{5 8 . 6 1}$ \\
\hline
\end{tabular}

The second dataset used in this study was collected by our group jointly with the Education Services for the Deaf and Blind (ESDB) of the North Carolina Department of Public Instruction under IRB 14046 [33], hence this dataset will be referred as ESDB dataset $[34]^{2}$ For the ESDB data, the 14 sessions were grouped into pairs to ease a granular analysis, hence ending with 7 sessions. The characteristics of each dataset can be found in Table \with the details of ESDB described in Table IIII In this work, the data coming from the wrist was not used since the performance obtained with it was not promising, as observed in our previous work [33]. Note that since this dataset has only one subject, it can be used to analyze the performance of a personalized model rather than a population-level model.

The ESDB dataset was collected using a Raspberry Pi Model 3, equipped with a touch-screen display to aid labelling the collected data in real-time. The Mbientlab's MetaMotionR IMU $3^{3}$ were utilized. The IMU device contains an accelerometer, gyroscope, LED and a piezoelectric vibration generator. The software application has a UI for real-time data labelling implemented in Python 3.6. The operating system is the Rasbpian Stretch. The IMU streams data to the embedded system over bluetooth. The IMU sampling rate is $100 \mathrm{~Hz}$. If the system were to be used for notifications, once a detection occurs, the

\footnotetext{
${ }^{1}$ http://cbslab.org/smm-dataset/

${ }^{2}$ https://doi.org/10.5281/zenodo.5559169

3 http://https//mbientlab.com/tutorials/MetaMotionR.html
} 
TABLE III: ESDB dataset details

\begin{tabular}{l|r|r|} 
& \multicolumn{2}{c}{ ESDB } \\
\cline { 2 - 3 } & Activity \% & Length (min) \\
\hline Sess. 1 & 66.14 & 84.85 \\
Sess. 2 & 37.05 & 44.89 \\
Sess. 3 & 31.23 & 41.00 \\
Sess. 4 & 10.25 & 36.38 \\
Sess. 5 & 62.10 & 52.28 \\
Sess. 6 & 80.73 & 56.87 \\
Sess. 7 & 53.33 & 46.61 \\
Sess. 8 & 68.46 & 62.64 \\
Sess. 9 & 68.46 & 62.64 \\
Sess. 10 & 81.83 & 26.88 \\
Sess. 11 & 74.46 & 71.78 \\
Sess. 12 & 33.96 & 32.14 \\
Sess. 13 & 74.84 & 29.48 \\
Sess. 14 & 56.21 & 55.99 \\
\hline Average & $\mathbf{5 7 . 0 7}$ & $\mathbf{5 0 . 3 2}$ \\
\hline
\end{tabular}

vibration generator in the MetaMotionR device could be activated. A picture of the data collection procedure is shown in Fig. 1

\section{B. Bayesian Neural Networks}

Deep learning has shown incredible performance in different applications but it is still hard to analytically understand the internal nature of such models, which paradoxically may prevent further advancement of this technology. Model uncertainty has been used as a way to evaluate such models offering a probabilistic interpretation of model's intrinsic factors driving its performance. In particular, techniques such as dropout have been used to capture variability in deep learning models in a similar way to ensemble learning by randomly removing some network connections during training time [35].

For a while, it has been known that an infinite-depth neural network (NN) with a distribution established over its weights converges to a Gaussian process [13], [35], [36], while finite approximation to weights distributions has been attempted under the framework of Bayesian Neural Networks [13], [14]. The authors in [15] have shown that a neural network with arbitrary depth and non-linearities with dropout before its weights, as normally used in NNs, is a Bayesian approximation of a Gaussian process marginalized over its covariance parameters. This allows the characterization of the uncertainty due to intrinsic parameters to the model and due to its input data. Next, a brief introduction of the method used by [15] is presented, which shows how model uncertainty can be characterized while enabling model interpretability.

Let us consider the estimated output $\hat{y}_{i}$ of a $\mathrm{NN}$ and the ground truth $y_{i}$ for an input $x_{i}$ with $i=1, \cdots, N$ where each data point $\left(x_{i}, y_{i}\right)$ comes from the dataset $(X, Y)$, i.e., the sets of input and output respectively. For our discussion, we will consider a NN with $L$ layers of the form:

$$
\hat{y}(x ; W)=\frac{1}{\sqrt{K_{L-1}}} W_{L} \cdot a\left(\cdots \frac{1}{\sqrt{K_{1}}} W_{2} \cdot a\left(W_{1} x+b_{1}\right)+b_{2} \cdots\right)
$$

where $a(\cdot)$ is some activation function, $W_{l} \in \mathbb{R}^{K_{l} \times K_{l-1}}$ are the $\mathrm{NN}$ weights and $b_{l} \in \mathbb{R}_{l}^{K}$ the vector of biases for each layer $l=1, \cdots, L$. A standard cost function often used for training of these networks (even when considering dropout) has the form:

$$
\mathcal{L}_{\text {std }}=\frac{1}{N} \sum_{i=1}^{N} E\left(y_{i}, \hat{y}_{i}\right)+\sum_{l=1}^{L} \lambda_{W, l}\left\|W_{l}\right\|_{2}^{2}+\sum_{l=1}^{L-1} \lambda_{b, l}\left\|b_{l}\right\|_{2}^{2}
$$

where $E(\cdot, \cdot)$ is a loss function, and the $\lambda$ 's are weight factors for $L_{2}$ regularization.

A deep Gaussian process (GP) is a model resulting of the hierarchical composition of GPs. A Gaussian process models a finite collection of its variables using a multivariate normal distribution with a defined covariance matrix function. Hence for a GP, the covariance matrix can be approximated using a variational distribution over each component of its spectral decomposition [15]. It is known that each hidden layer in a NN can be represented by one of the layers of a deep GP [15].

In this context, the predictive distribution of the deep GP model can be represented as:

$$
\begin{aligned}
P(y \mid x, X, Y) & =\int P(y \mid x, w) P(w \mid X, Y) d w \\
P(y \mid x, w) & =\mathcal{N}\left(y ; \hat{y}(x, w), \tau^{-1} I_{D}\right)
\end{aligned}
$$

for some precision hyper-parameter $\tau>0$, where $\mathcal{N}(y ; \mu, \Sigma)$ represent the normal Gaussian distribution with mean $\mu$ and covariance $\Sigma$, and $X$ and $Y$ are the training set. Since the posterior $P(w \mid X, Y)$ is intractable, the authors in [15] shows how 
to approximate it using variational inference. Such approximation is done by Monte Carlo Dropout sampling [15] and by minimizing the KL divergence between an approximating distribution $q(w)$ and $P(w \mid X, Y)$, as it will be shown next.

Now, let $W_{l}=M_{l} \cdot \operatorname{diag}\left(\left[z_{l, j}\right]_{j=1}^{K_{l}-1}\right)$, with $z_{l, j} \approx \operatorname{Bernoulli}\left(p_{l}\right)$ for $l=1, \cdots, L$, and $j=1, \cdots, K_{l-1}$, given some matrices $M_{l}$ and probability $p_{l}$ as variational parameters. Note, that by using this argument we are in practice sampling the elements of $M_{l}$. Let $q(w)$ be the distribution over the matrix $M_{l}$. We use the KL divergence between $q(w)$ and the posterior $P(w \mid X, Y)$ as our objective for minimization, which (after some mathematical manipulation) can be expressed as:

$$
-\int q(w) \log P(Y \mid X, w) d w+K L(q(w) \| P(w)) \text {. }
$$

Minimizing equation 4 is equivalent to maximizing the log evidence lower bound [37], where the first term is equivalent to $-\sum_{i=1}^{N} \int q(w) \log P\left(y_{i} \mid x_{i}, w\right) d w$. Note that equation 4 would require integration over the entire space for the variable $w$, which does not scale well [38]. Therefore, it is shown next how the integral can be effectively approximated.

As shown in [15], [39], the Monte Carlo approximation of the two terms in equation (4) for the deep GP considered gives:

$$
\mathcal{L}_{\mathrm{GP}-\mathrm{MC}} \propto-\frac{1}{N} \sum_{i=1}^{N}-\log P\left(y_{i} \mid x_{i}, w_{i}\right)+\sum_{l=1}^{L}\left(\frac{p_{l}}{2 \tau N}\left\|M_{l}\right\|_{2}^{2}+\frac{1}{2 \tau N}\left\|b_{l}\right\|_{2}^{2}\right)
$$

where $w_{i}$ are sampled from the distribution specified by $q(w)$ by obtaining realizations of the Bernoulli distribution $z_{l, j}$ as it is done during the dropout process. By setting $\left.E\left(y_{i}, \hat{y}_{i}\left(x_{i} ; w_{i}\right)\right):=-\log P\left(y_{i} \mid x_{i}, w_{i}\right)\right)$, we get an expression with similar form to equation 2 .

For the case of regression, and given enough training data (so the terms due to regularization of the weights and biases is negligible), we can approximate equation (5] by [40]:

$$
\mathcal{L}_{\text {reg }}=\frac{1}{N} \sum_{i=1}^{N}\left[\frac{1}{2} \hat{\sigma}_{i}^{-2}\left\|y_{i}-\hat{y}_{i}\right\|^{2}+\frac{1}{2} \log \left(\hat{\sigma}_{i}^{2}\right)\right],
$$

where $\hat{\sigma}_{i}$ is a variable that captures the observation noise for sample $\left(x_{i}, y_{i}\right)$ which is treated as another output of the NN.

Next, we discuss how to approximate the predictive distribution given a new sample point $x^{*}$ once the model has been trained. The distribution for the predicted value $y^{*}$ is [39]:

$$
q\left(y^{*} \mid x^{*}\right)=\int P\left(y^{*} \mid x^{*}, w\right) q(w) d w .
$$

This distribution is approximated using a moment-matching technique by finding an estimate for the first two moments with the help of Monte-Carlo integration. The first moment approximation is obtained by the following:

$$
\mathbb{E}_{q\left(y^{*} \mid x^{*}\right)}\left(y^{*}\right) \approx \frac{1}{T} \sum_{t=1}^{T} \hat{y}^{*}\left(x^{*}, w^{t}\right)
$$

where $\left\{w^{t}\right\}_{t=1}^{T}$ are obtained by drawing $T$ samples from the distribution specified by $q(w)$. This expression is basically $T$ averaged forward stochastic passes through the NN.

The second moment approximation is obtained by:

$$
\mathbb{E}_{q\left(y^{*} \mid x^{*}\right)}\left(y^{* \top} y^{*}\right) \approx \tau^{-1} I_{D}+\frac{1}{T} \sum_{t=1}^{T} \hat{y}^{*}\left(x^{*} ; w^{t}\right)^{\top} \hat{y}^{*}\left(x^{*} ; w^{t}\right)
$$

Hence the model's predictive variance is obtained by:

$$
\operatorname{Var}_{q\left(y^{*} \mid x^{*}\right)}\left(y^{*}\right) \approx \tau^{-1} I_{D}+\frac{1}{T} \sum_{t=1}^{T} \hat{y}^{*}\left(x^{*} ; w^{t}\right)^{\top} \hat{y}\left(x^{*} ; w^{t}\right)-\mathbb{E}_{q\left(y^{*} \mid x^{*}\right)}\left(y^{*}\right)^{\top} \mathbb{E}_{q\left(y^{*} \mid x^{*}\right)}\left(y^{*}\right)
$$

which is the same as the sample variance of $T$ forward passes through the NN plus the inverse of the model's precision.

There are two types of uncertainties considered for quantification [38] (1) aleatoric uncertainty, which is the uncertainty associated with the data, (2) epistemic uncertainty, which is the uncertainty associated with the model [41] and usually can be explained away with enough data [42]. For aleatoric uncertainty, there are two subtypes: heteroscedastic and homoscedastic. The first quantity is dependent on the data, while the second one assumes identical noise for all input samples.

Predictive entropy [43] measures the amount of uncertainty associated with a measurement. With the Monte Carlo dropout sampling, it is approximated as

$$
H\left[y^{*} \mid x^{*}, X, Y\right]=-\sum_{c^{\prime}}\left(\frac{1}{T} \sum_{t=1}^{T} P\left(y^{*}=c^{\prime} \mid x, w^{t}\right)\right) \log \left(\frac{1}{T} \sum_{t=1}^{T} P\left(y^{*}=c^{\prime} \mid x, w^{t}\right)\right)
$$


Mutual information (MI) between the posterior over the weights and the prediction $y^{*}$ quantifies the uncertainty in the BNN's output [44]. This measure is larger when the stochastic predictions are less stable, and it is calculated via:

$$
I\left[y^{*} \mid x^{*}, X, Y\right]=H\left[y^{*} \mid x^{*}, X, Y\right]+\frac{1}{T} \sum_{c^{\prime}, t}\left[P\left(y^{*}=c^{\prime} \mid x, w^{t}\right) \cdot \log \left(P\left(y^{*}=c^{\prime} \mid x, w^{t}\right)\right)\right] .
$$

Predictive entropy represents the effect of epistemic and aleatoric uncertainties. On the other hand, mutual information is a representation of the epistemic model uncertainty [41].

The regression aleatoric uncertainty is now extended to a classification task, by modelling the regression uncertainty of the logit vector - the output of the last layer before the Softmax activation function. A Gaussian distribution is placed over the logit vector as $\hat{z} \sim \mathcal{N}\left(y, \sigma^{2}\right)$, where $\left[\hat{y}, \sigma^{2}\right]=f^{W}(x)$ with $f^{W}$ as the NN. The expected log likelihood for each training sample is described as [45]:

$$
\mathcal{L}=\log \left\{\mathbb{E}_{\mathcal{N}\left(\hat{z} ; y, \sigma^{2}\right)}\left[\operatorname{Softmax}(\hat{z})_{c}\right]\right\},
$$

where $y$ is the ground truth label of $x$ and $c$ is the index for the ground truth label.

Since equation 13 is analytically intractable, it is approximated by Monte Carlo integration. Denote $\hat{z}^{t}=f^{W}(x)+\sigma \cdot \epsilon^{t}$, where $\epsilon^{t}$ follows a standard Gaussian distribution. The loss function becomes

$$
\mathcal{L}=\log \frac{1}{T} \sum_{t=1}^{T} \exp \left[\hat{z}_{c}^{t}-\log \sum_{c^{\prime}} \exp \left(\hat{z}_{c^{\prime}}^{t}\right)\right]
$$

where $T$ is the number of Monte Carlo sampling iterations and $c^{\prime}$ is the class index of the logit vector $\hat{z}$ [45]. The loss function in equation (14) is the one that is going to be used later on in the experiments.

The uncertainty quantification relies on the estimation of the approximated predictive probability which for the classification case is shown by [42] as the output of softmax vector:

$$
p^{*} \approx \frac{1}{T} \sum_{t=1}^{T} \operatorname{Softmax}\left[\hat{y}^{*}\left(x^{*} ; w^{t}\right)\right]
$$

Although, there are claims in the literature showing that the deep learning architectures have so much capacity that they can even fit corrupted data [46], we intend to show that the Bayesian approach does bring improvement to the predictions. Although we do not explore the impact of the prior in the predictions, we recognize its crucial role for accurate estimations of the posterior, as shown by [47]-[49] and many others.

\section{Probability Calibration}

Assume a multi-class classifier with a prediction and corresponding predicted probability $[\hat{y}, \hat{\rho}]=\mathcal{H}(x)$ for an input $x$ with $\hat{\rho}$ representing the probability that the class label is correct. In this case, we would expect $\hat{\rho}$ to match the empirical probability of this event. That is, a sufficient condition for calibration can be defined as

$$
P(\hat{y}=y \mid \hat{\rho}=\rho)=\rho, \quad \forall \rho \in[0,1] .
$$

It is known that the predicted probability is not calibrated for neural networks, especially in case of BNNs [38]. In this work, we employ the approach of Zhong et al. [16], [50] of utilizing the three uncertainties obtained with the BNN framework (i.e., the variance estimate of the prediction, the entropy and the mutual information) to find a map, say $\Psi$, from the uncertainties domain to a calibrated probability domain. Hence, a calibration function $\Psi: \mathbb{R}^{3} \rightarrow[0,1]$ is desired such that $\hat{\rho}=\Psi \circ U$ produces calibrated probabilities, where $U$ represents the three uncertainty measures from the BNN. In our framework, we used a neural network to approximate $\Psi$ with architecture composed of 3 fully connected layers (FCN) with 32 (activation tanh) and 64 (activation tanh) neurons in the hidden layers and one neuron (activation sigmoid) for the output layer.

\section{The Models}

The Bayesian deep learning (DL) uncertainty quantification approach is applied to an end-to-end model, which together with Sadouk et al [28] represents the state of the art for body-rocking detection in our assessment. We consider Rad et al. [27] as our baseline, which used a fully end-to-end deep learning approach for the same goal. For this implementation, we re-used most of the CNN pipeline developed by them 4 This model is deeper than the one from [28] with 3 CNN layers of 4, 4 and 8 kernels respectively. Each of these layers has filter sizes of 10 and stride of 1 , the output of the CNN is flattened and passed through a batch normalization layer followed by an FCN with 8 nodes, the logits are then dropped out with $p=0.2$ to generate a hot encoding output for body-rocking and no-body-rocking status. For training this network, a cross-entropy loss function is used. A diagram of Rad's architecture is shown in Fig. 2(a). This model will be referred as Rad's model or Rad's approach and it represents the state of the art for end-to-end detection of body-rocking in our assessment.

${ }^{4}$ https://gitlab.fbk.eu/MPBA/smm-detection 
For a fair use of Bayesian DL framework, we evaluate an additional model that is wider than the one aforementioned. The model will be referred as WiderNet which is basically an upscaled version of Rad's approach in terms of number of filters per layer. For instance, the model WiderNet $2 x$ would be a 3 layer CNN model, exactly as Rad's approach, but with 8,8 and 16 filters per layer of size 10 each. Two variants of models are created differing only in the amount of nodes in their FCN. Therefore, the model "WiderNet 2x, FCN 128" has 128 nodes in its FCN as one can see in Fig. 2 b), thus the abbreviation "FCN" will be followed by the quantity of nodes. The use of a WiderNet is justified since Rad's model is a narrow model (few filters per layer) and may not display the advantages of the Bayesian approach since Rad's shallower aspects do not contribute to the premises of Bayesian formulation, namely, the deeper/wider the model, the closer to a Gaussian process. In order to show that wider models gain more benefits when using Bayesian approach, the WiderNet will be upscaled and evaluated in $2 \mathrm{x}, 4 \mathrm{x}, 8 \mathrm{x}$ and 16x for comparison. Each upscaled version is going to be evaluated with the original Rad's FCN (i.e., 8 nodes) and 128 nodes. Implementation is publicly available 5

\section{E. Dataset Pre-Processing and Evaluation Strategy}

As mentioned earlier, we used the public EDAQA dataset and our ESDB dataset for analysis.

The models are evaluated in both datasets with the leave-one-subject-out (for the EDAQA dataset) or leave-onesession-out (for the ESDB dataset) strategies for training and testing. The training was performed for 45 epochs. For each subject left out, the mentioned procedure is repeated 10 times to account for additional variability in the training process.

For all models, the data was filtered by a Butterworth band-pass filter, segmented into windows of $N$ samples for each axis (either 3 axes of gyro of accelerometer measurements), and trained end-to-end as described in their original work [27]. The window size is a moving window with $N=10$.

For the Bayesian approach, the models had their original loss function replaced by the Bayesian loss (see equation (14)), and dropout is added for each layer with probability 0.05. A diagram showing the additions of the Bayesian components to Rad's model can be observed in Fig. 2(c).

1) Transfer learning for model improvement: As comprehensively explored in statistics and DL literature [27], [28], transfer learning (TF) is useful to take advantage of data coming from similar domains, consequently increasing model generalization capabilities. In the application of wearable sensors, transfer learning is especially useful to allow a model to work with sensors placed in different body limbs.

In our particular scenario, we initially train the best performing model using the EDAQA data and later on we re-train the model on the ESDB dataset conserving most of its parameters. As explained before, the torso is the optimal location for body-rocking, since it has minimal coupling with other repetitive activities that could be performed by other body limbs. On the other hand, data coming from the arm has significant mechanical coupling with other activities, which makes it much more challenging to work with. For example, in a classroom environment, repeatedly grabbing folders from the student's backpack to their desk would trigger a detector on the wrist but it may not on the chest. The transfer learning technique has been chosen as an aid to improve the model's performance in the challenging (ESDB) arm data set.

2) Uncertainty quantification as a criterion for choosing reliable predictions: We make sure of the uncertainty quantification metrics as well as the calibrated probabilities generated by the Bayesian DL framework to develop a criterion to establish whether a prediction made by the model should generate a notification or not. More specifically, we will make use of a threshold on these quantities as a criterion for selection of a reliable detection. As mentioned in the introduction, excessive false positives can lead to alert fatigue. The trade-off between the distributions of correct and incorrect predictions will be analyzed as the criterion is changed.

5 https://github.com/rafa-coding-projects/Body-Rocking 
3) Metrics: For the evaluation metrics we focus on Area-Under-Curve (AUC) of the computed Receiver Operating Characteristic (ROC) curve and precision. The reason for AUC is for it being known as less sensitive to oscillations in predictions and a good indicator for generalization, in contrast to other metrics such as accuracy. The other metric, precision, is defined as:

$$
\text { precision }=\frac{T P}{T P+F P}
$$

with $T P$ and $F P$ being true positives and false positives respectively. Lastly, we also compute F1-score which is defined as:

$$
F 1=2 \times \frac{\text { precision } \times \text { recall }}{\text { precision }+ \text { recall }}
$$

where recall is defined as,

$$
\text { recall }=\frac{T P}{T P+F N}
$$

for FN being false negatives. For completeness, accuracy is also computed but left in Table IV, Table V, Table VI. The metrics above are calculated using the model output specified by (15) when using the Bayesian approach, and the regular softmax output (as shown in Fig. 2 a and b) for non-Bayesian models.

\section{Results}

00. The models are evaluated using the EDAQA and ESDB datasets. In Fig. 3, we observe the distinct performance of each approach on the datasets.

TABLE IV: Performance on EDAQA dataset, Study 1

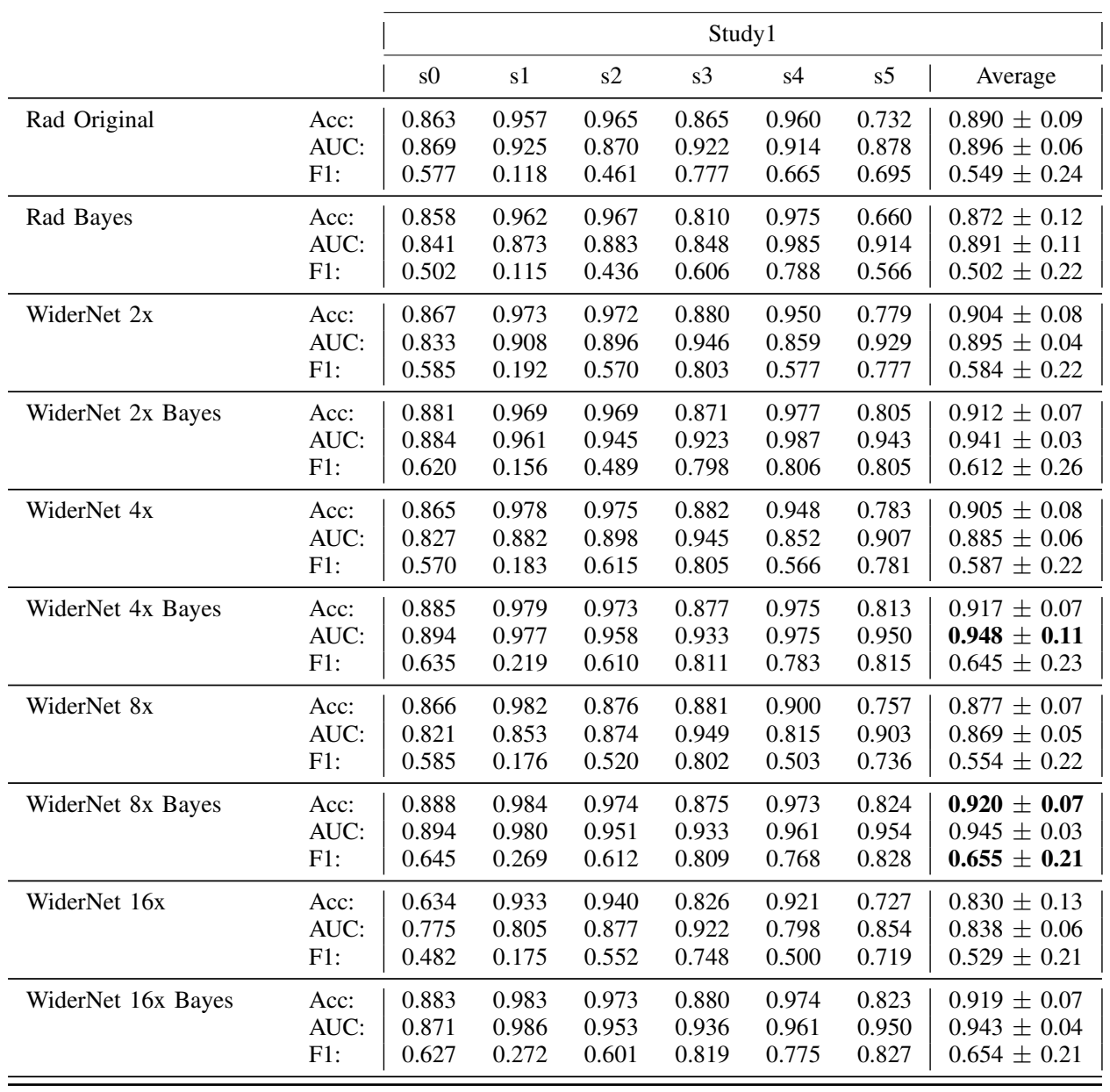




\section{AUC Score}
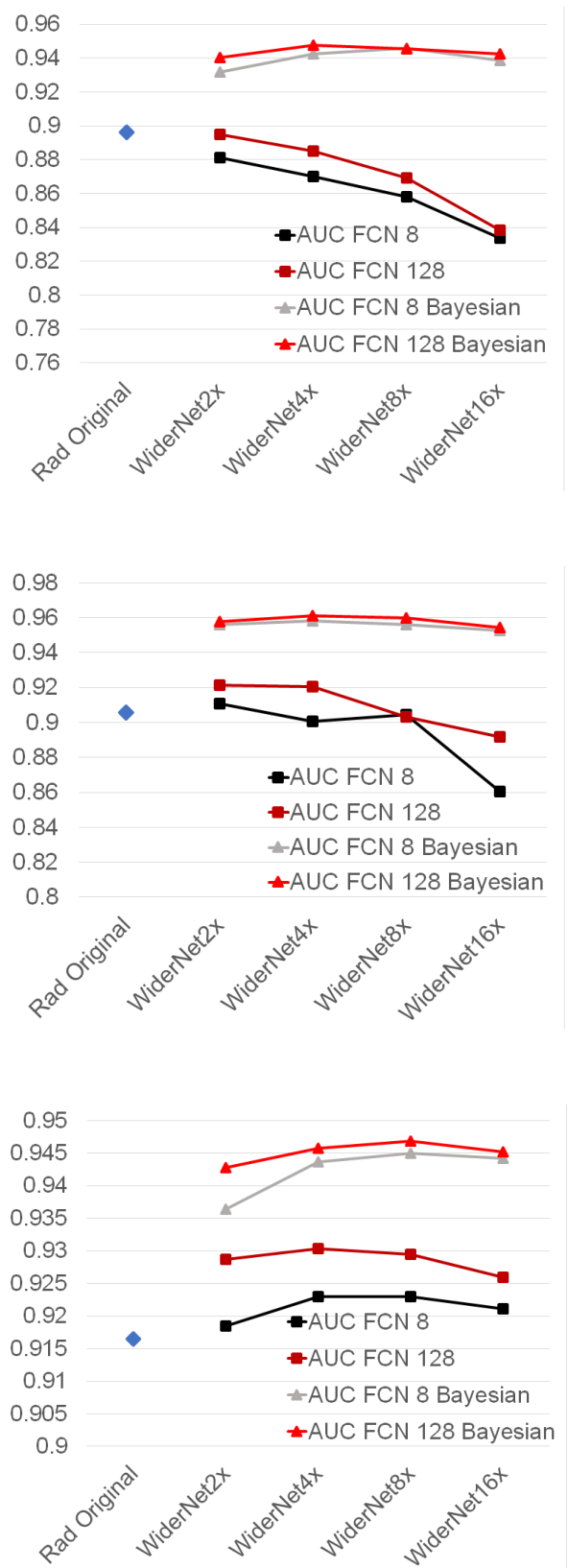

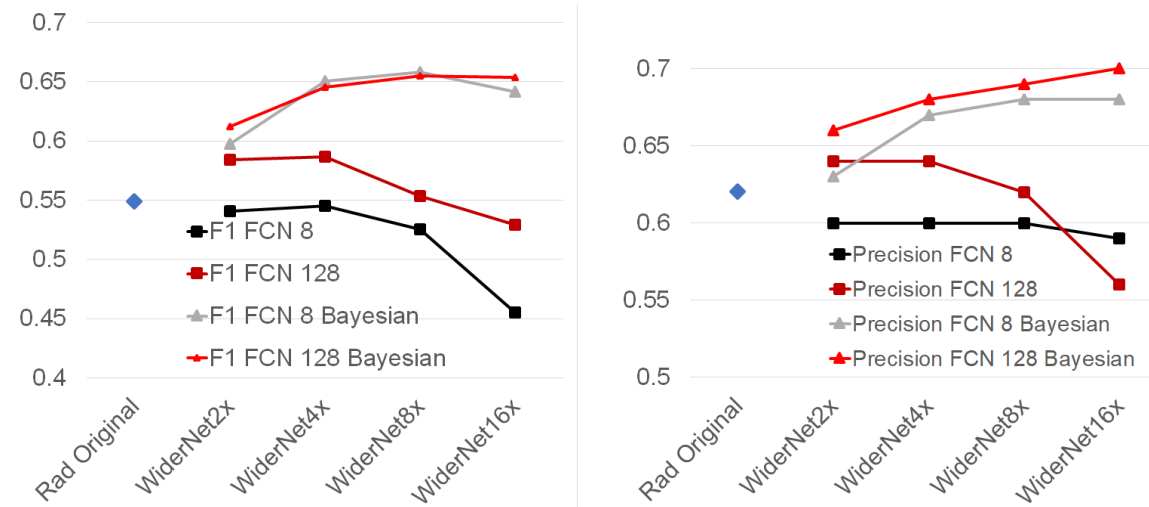

(a) EDAQA dataset, study 1.
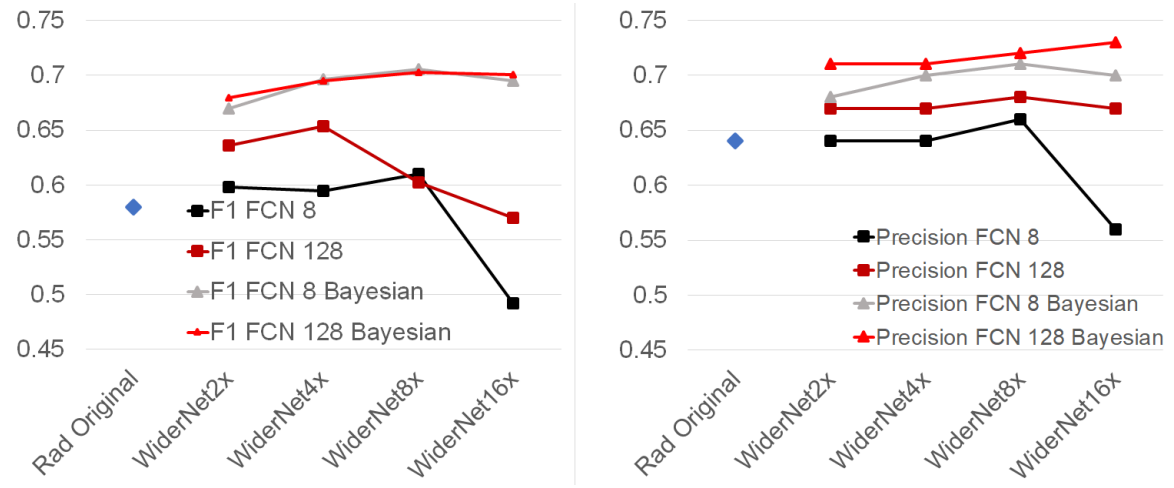

(b) EDAQA dataset, study 2.
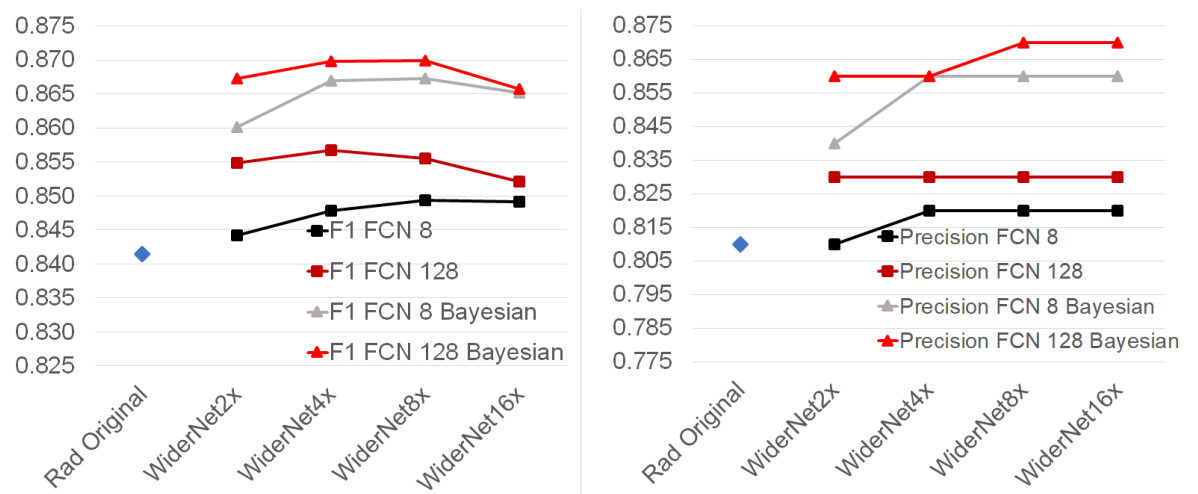

(c) ESDB dataset.

Fig. 3: Performance of the presented architectures on the datasets. The architectures are evaluated under different metrics to aid the elicitation of Bayesian approach on a regular CNN. The columns represent the averages of AUC (left), F1-score (middle) and precision (right), across all subjects and 10 runs. Rad Original is the baseline represented by a blue dot, while the legends identifies the WiderNet variants. We observe an improvement in all models when considering their Bayesian variant. This improvement is more noticeable in the ESDB dataset. Best viewed in color.

\section{A. Bayesian Approach Compared to Current Methods}

For EDAQA Study 1 in Fig. 3 (a), one can observe that the AUC, and F1-score obtained by Rad's approach is in general superior to the WiderNet models without a Bayesian approach (with a legend as "AUC FCN 8" and "AUC FCN 128"). By evaluating the curves for the AUC plot, one can observe that increased model complexity seems to be degrading model's performance, most likely due to overfitting. The Bayesian approach stands out as being superior to Rad's approach by almost one standard deviation when using WiderNet 8x FCN 128. The figure also shows that the Bayesian approach does not require an aggressive increase on capacity of the architecture in order to perform better since WiderNet 2x already increases the AUC 
by $3 \%$, all obtained AUC values are around $94 \%$ for the Bayesian approach.

We continue evaluating Study 1, but now for F1-score in Fig. 3 (a) in the middle column. As expected, the performance degradation due to increase in capacity of non-Bayesian models is also reflected in their F1-score. Also, different improvements are obtained as the WiderNet's capacity is increased, which is more noticeable than when analyzing the AUC. The F1-score for Bayesian approach increases from $61 \%$ to almost $66 \%$. WiderNet 8x provided the highest F1-score of $65.8 \%$, just slightly above WiderNet 8x FCN 128 with $65.5 \%$, more than 10\% compared to the F1-Score obtained with Rad's approach of 54.9\%. Precision is also further improved by the Bayesian approach from $62 \%$ with Rad's model to reaching up to $70 \%$ with WiderNet 16x FCN 128. Therefore, for F1-score and precision, the Bayesian approach provided greater improvement than for AUC.

For EDAQA Study 2, the reader can refer to Fig. 3 b). The performance in this portion of EDAQA dataset is superior than the performance obtained in Study 1 as also shown in [28]. The same model degradation trend when increasing capacity observed in Study 1 is also present in Study 2 when considering non-Bayesian approaches. On the other hand, the WiderNet 8x provided an F1-score of $70 \%$ compared to $58 \%$ of Rad's model, an improvement of $12 \%$. The precision when using WiderNet 16x was of $73 \%$ and Rad's model presented $68 \%$, an improvement of $5 \%$.

TABLE V: Performance on EDAQA dataset, Study 2

\begin{tabular}{|c|c|c|c|c|c|c|c|c|}
\hline & & \multicolumn{7}{|c|}{ Study2 } \\
\hline & & s0 & s1 & $\mathrm{s} 2$ & s3 & s4 & s5 & Average \\
\hline \multirow{3}{*}{$\begin{array}{l}\text { Rad } \\
\text { Original }\end{array}$} & Acc: & 0.863 & 0.962 & 0.967 & 0.921 & 0.958 & 0.767 & $0.906 \pm 0.08$ \\
\hline & AUC: & 0.877 & 0.907 & 0.881 & 0.965 & 0.911 & 0.894 & $0.906 \pm 0.08$ \\
\hline & F1: & 0.563 & 0.130 & 0.533 & 0.884 & 0.643 & 0.727 & $0.580 \pm 0.25$ \\
\hline \multirow{3}{*}{$\begin{array}{l}\text { Rad } \\
\text { Bayes }\end{array}$} & Acc: & 0.868 & 0.960 & 0.967 & 0.861 & 0.981 & 0.652 & $0.881 \pm 0.12$ \\
\hline & AUC: & 0.866 & 0.931 & 0.945 & 0.904 & 0.991 & 0.884 & $0.920 \pm 0.05$ \\
\hline & F1: & 0.538 & 0.098 & 0.463 & 0.740 & 0.839 & 0.502 & $0.530 \pm 0.26$ \\
\hline \multirow[t]{3}{*}{ WiderNet $2 \mathrm{x}$} & Acc: & 0.874 & 0.982 & 0.974 & 0.913 & 0.960 & 0.833 & $0.923 \pm 0.06$ \\
\hline & AUC: & 0.866 & 0.938 & 0.924 & 0.961 & 0.899 & 0.941 & $0.921 \pm 0.03$ \\
\hline & F1: & 0.610 & 0.234 & 0.615 & 0.869 & 0.646 & 0.840 & $0.636 \pm 0.23$ \\
\hline \multirow[t]{3}{*}{ WiderNet 2x Bayes } & Acc: & 0.890 & 0.974 & 0.979 & 0.899 & 0.981 & 0.858 & $0.930 \pm 0.05$ \\
\hline & AUC: & 0.905 & 0.972 & 0.969 & 0.949 & 0.992 & 0.958 & $0.958 \pm 0.03$ \\
\hline & F1: & 0.660 & 0.203 & 0.660 & 0.854 & 0.833 & 0.866 & $0.679 \pm 0.25$ \\
\hline \multirow[t]{3}{*}{ WiderNet $4 x$} & Acc: & 0.883 & 0.986 & 0.978 & 0.919 & 0.955 & 0.808 & $0.921 \pm 0.07$ \\
\hline & AUC: & 0.864 & 0.961 & 0.919 & 0.966 & 0.880 & 0.933 & $0.920 \pm 0.08$ \\
\hline & F1: & 0.662 & 0.288 & 0.664 & 0.876 & 0.618 & 0.812 & $0.653 \pm 0.20$ \\
\hline \multirow[t]{3}{*}{ WiderNet 4x Bayes } & Acc: & 0.894 & 0.982 & 0.978 & 0.900 & 0.978 & 0.864 & $\mathbf{0 . 9 3 3} \pm \mathbf{0 . 0 5}$ \\
\hline & AUC: & 0.913 & 0.982 & 0.967 & 0.954 & 0.988 & 0.962 & $0.961 \pm \mathbf{0 . 0 3}$ \\
\hline & F1: & 0.672 & 0.277 & 0.680 & 0.856 & 0.813 & 0.873 & $0.695 \pm 0.22$ \\
\hline \multirow[t]{3}{*}{ WiderNet $8 \mathrm{x}$} & Acc: & 0.871 & 0.973 & 0.970 & 0.910 & 0.955 & 0.739 & $0.903 \pm 0.09$ \\
\hline & AUC: & 0.839 & 0.904 & 0.908 & 0.962 & 0.885 & 0.920 & $0.903 \pm 0.03$ \\
\hline & F1: & 0.586 & 0.222 & 0.641 & 0.861 & 0.606 & 0.700 & $0.602 \pm 0.21$ \\
\hline \multirow[t]{3}{*}{ WiderNet 8x Bayes } & Acc: & 0.889 & 0.984 & 0.978 & 0.900 & 0.979 & 0.871 & $0.933 \pm 0.05$ \\
\hline & AUC: & 0.908 & 0.991 & 0.965 & 0.951 & 0.986 & 0.960 & $0.960 \pm 0.03$ \\
\hline & F1: & 0.649 & 0.329 & 0.682 & 0.855 & 0.821 & 0.880 & $0.703 \pm 0.21$ \\
\hline \multirow[t]{3}{*}{ WiderNet $16 x$} & Acc: & 0.858 & 0.954 & 0.976 & 0.850 & 0.927 & 0.721 & $0.881 \pm 0.09$ \\
\hline & AUC: & 0.811 & 0.930 & 0.920 & 0.928 & 0.869 & 0.893 & $0.892 \pm 0.05$ \\
\hline & F1: & 0.499 & 0.256 & 0.665 & 0.774 & 0.547 & 0.681 & $0.570 \pm 0.18$ \\
\hline \multirow[t]{3}{*}{ WiderNet 16x Bayes } & Acc: & 0.890 & 0.987 & 0.978 & 0.899 & 0.978 & 0.860 & $0.932 \pm 0.05$ \\
\hline & AUC: & 0.899 & 0.990 & 0.951 & 0.949 & 0.975 & 0.961 & $0.954 \pm 0.03$ \\
\hline & F1: & 0.657 & 0.356 & 0.660 & 0.853 & 0.808 & 0.869 & $0.700 \pm 0.19$ \\
\hline
\end{tabular}

Finally for ESDB in Fig. 3.c), one can observe a superior average performance by WiderNet variants in general when compared to Rad's model. This happens although all AUC values are within a range of about 4\%. Thus, considering AUC, one can see that widening Rad's model provided improvements for the non-Bayesian approach of WiderNet 2x and 4x only. Increasing the model capacity any further degrades the AUC, as the non-Bayesian models variants WiderNet 8x and WiderNet 16x show, independently of how many nodes are placed in the FCN. The Bayesian approaches had an even higher performance since AUC improvements were observed until an increase in capacity by $8 \mathrm{x}$. The models that had FCN 128 seem to be less sensitive to capacity increase. The FCN 128 Bayesian variants, seem to have plateau in terms of performance, leading us to believe that the framework has reached a limit in performance. Applying the Bayesian framework to Rad's model slightly improved its AUC score from 92\% to 93\% while the Bayesian WiderNet 8x had 95\%.

Finally, considering also AUC for model generalization as well as dealing with an imbalanced data, the up-scaled model WiderNet 8x FCN 128 seems to be the best performing one. In Fig. 4 one can verify how AUC, and precision play a role between the best models and Rad's. 


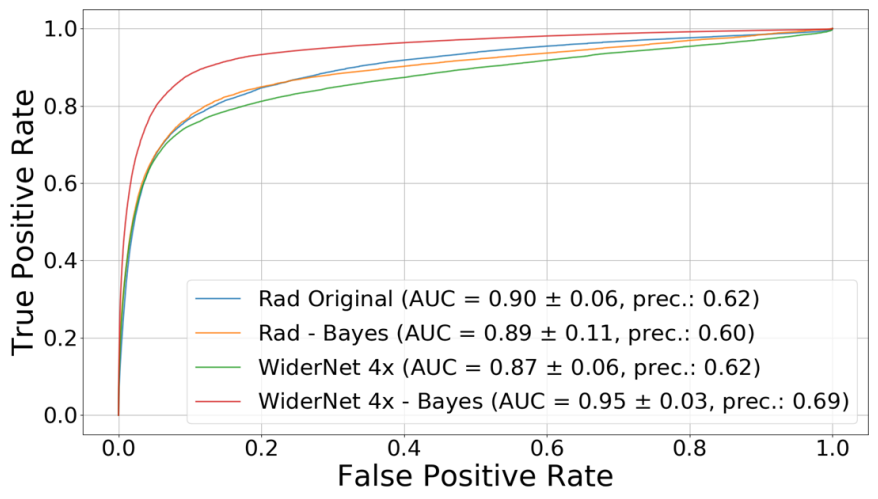

(a) EDAQA dataset, study 1

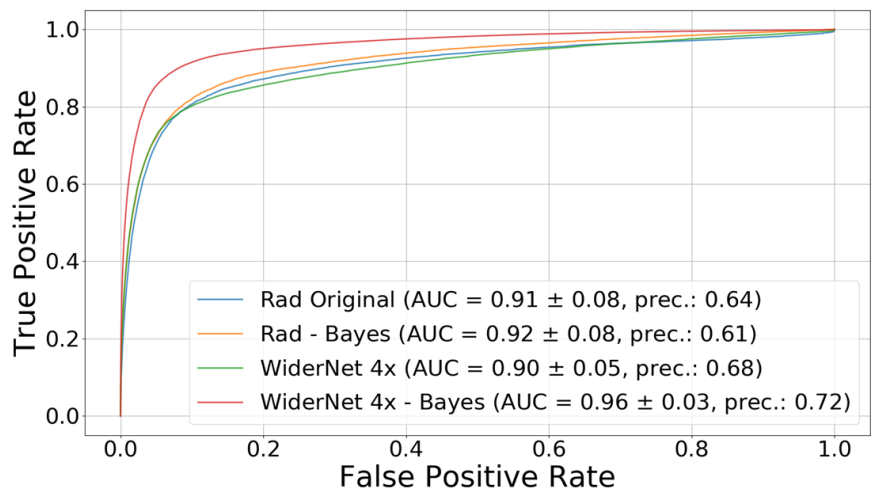

(b) EDAQA dataset, study 2

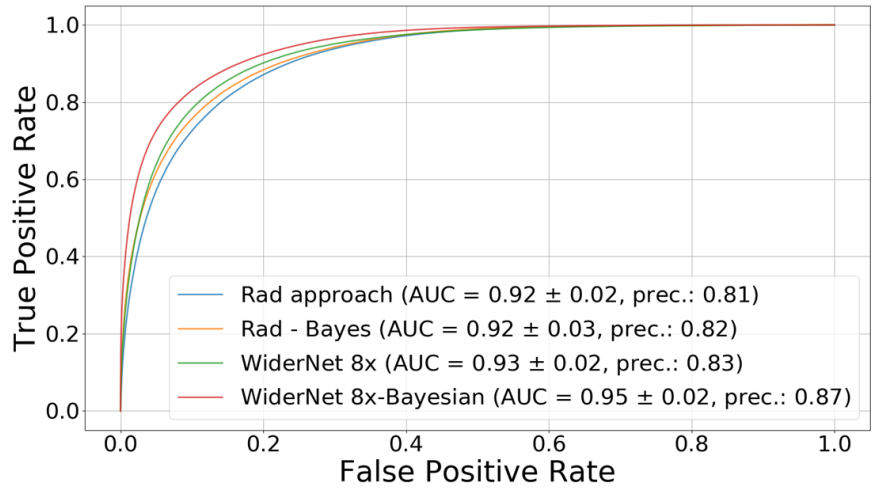

(c) ESDB dataset

Fig. 4: ROC curves for each dataset for Rad's model and WiderNet 8x, FCN 128 (i.e., with 128 neurons in their fully connected layer. The Bayesian model with the higher capacity performs best in all datasets. Best viewed in color.

\section{B. Effect of Transfer Learning (TF)}

We evaluated all Bayesian WiderNet FCN 128 variants using TF. It was observed that TF from the EDAQA to the ESDB dataset was not effective for Rad's approach, it rather substantially decreased their performance. We noticed that Bayesian WiderNet models obtained better performance with TF than Rad's model, but still slightly worse than training the models from scratch. It is important to note that [28] obtained good results and improvements by using TF from one subject to another. However, the same body limbs were being used while in our case, TF was attempted from torso data to right upper arm data. The sensing modalities are also different, since EDAQA dataset only uses accelerometer, whereas in ESDB the body-rocking activity is more evident in the gyroscope data.

The transfer learning is done by first training a Bayesian approach model from scratch on EDAQA Study 1, since from previous results, it seems to be a bit more challenging for the models than Study 2, the imbalance in that portion of EDAQA dataset is also less severe than in Study 2. Then, the first CNN layer and the FCN of the model are trained on ESDB data analogously as before, namely, with 35 epochs, using leave-one-subject out for the testing set and repeating this procedure 10 
TABLE VI: Performance on ESDB dataset

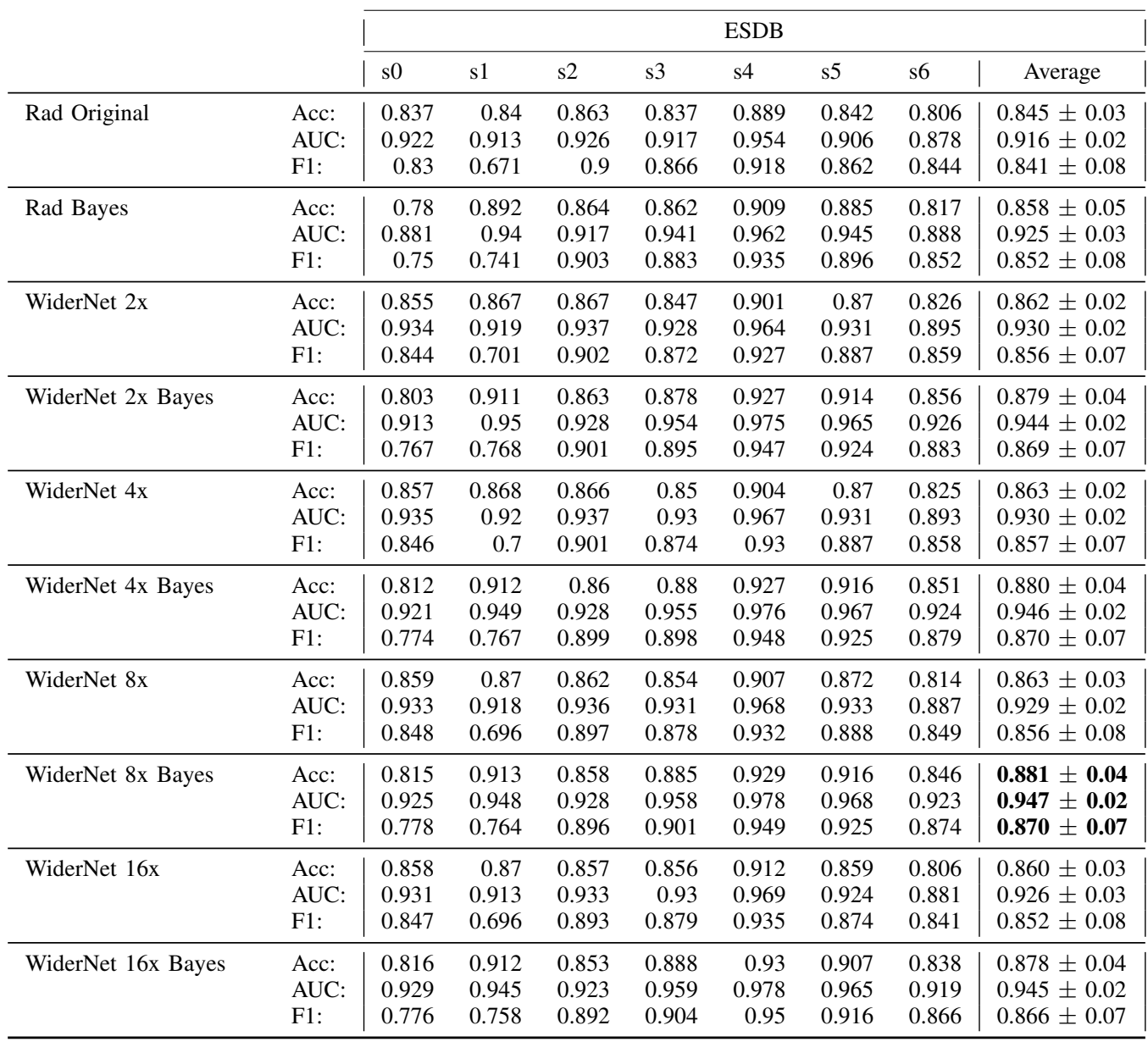

times for each subject.

We also analyzed the reliability plots or calibration diagrams, which capture the correctness of the calibrated probability $\rho$ (as described in section III-C). The supplemental material ${ }^{5}$ contains one example of a reliability plot. The $\mathrm{x}$-axis of the plots captures the mean calibrated probability value (i.e., which value of $\rho$ is predicted), and the y-axis corresponds to the fraction of positives in the dataset. Ideally, these numbers should match and the resulting curves should follow a diagonal line. In order to capture the offset from this ideal configuration, we report the Area From Diagonal (AFD), i.e., the area between the model curve and the diagonal. The AFD is reported for all WiderNet models with FCN 128 with and without TF in Fig. 5 We observe that TF seems to help the Bayesian models to neutralize the effect of higher variability as the model capacity increases.

\section{Uncertainty-based Detection Selection}

In this section, we explore the use of uncertainty as a criterion to select only predictions with high confidence. The Bayesian WiderNet 8x, FCN 128 model is used for this discussion since it is the model that provided the best overall performance among all models. The exploration is done in the ESDB dataset only, since this is the focus of this manuscript for developing real-time notification systems.

The distributions of the Entropy and MI measures of uncertainty for the correct and incorrect predictions are displayed in Fig. 6 One can observe that the distribution of incorrect predictions have a higher occurrence of entropy values greater than 0.4. One cannot tell much about a distinct pattern of MI since both groups have high concentration of values smaller than $10^{-1}$, therefore, only entropy is used for the analysis detailed next. Rad's model displays similar patterns.

We consider two uncertainty criteria for detection selection: (1) Setting positive detections with entropy above a specified threshold to be a negative (i.e., only keeping those detections with entropy that is low enough); and (2) Setting positive detections with calibrated probability below a specified threshold to be negative (i.e., only keeping those detection with calibrated probability that is high enough). Making use of the calibrated probability as a selection criteria is more desirable since the probability values can be more easily interpreted, and also because (as seen in the discussion below) it provides a 


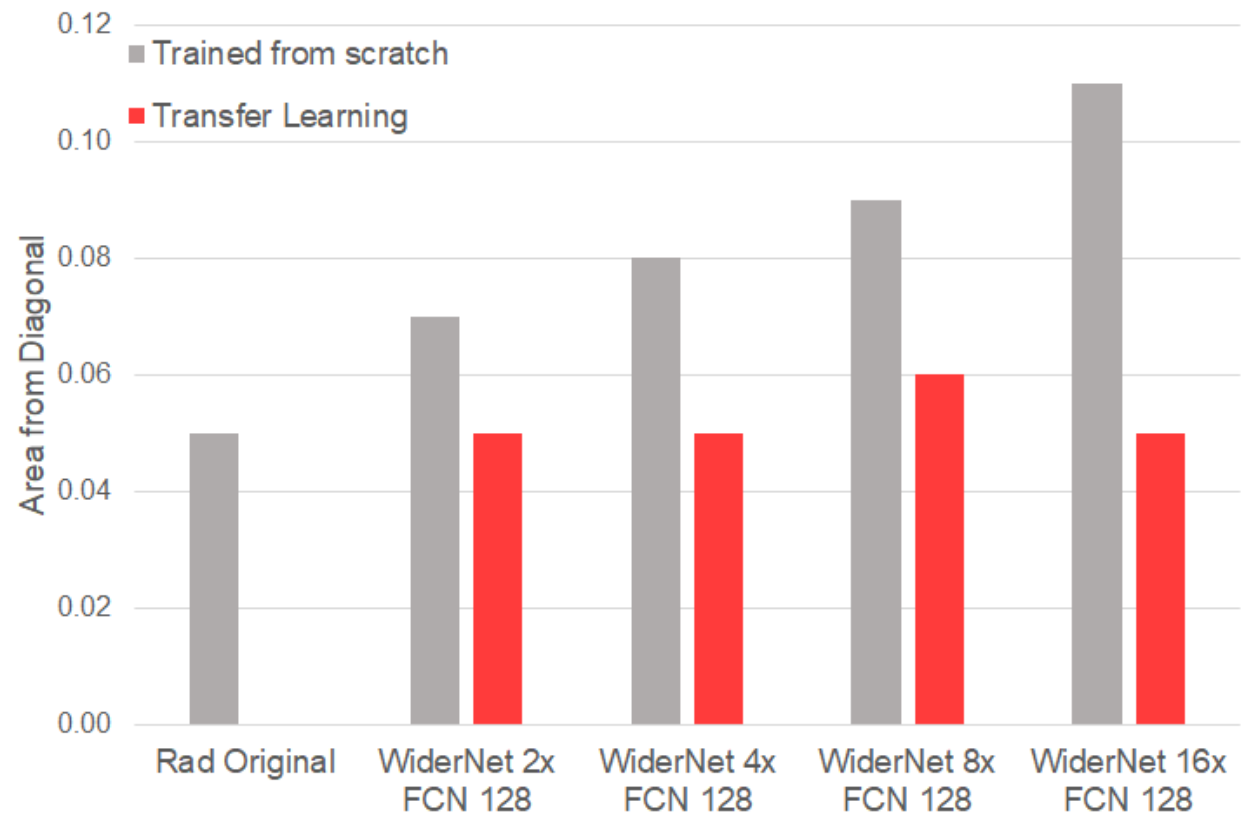

Fig. 5: Behavior of area from the diagonal of reliability plots with and without transfer learning (TF). TF prevents this error metric from increasing as the model capacity increases. Best viewed in color.

better trade-off than using a single uncertainty measure. The goal is to use these criteria for selection of prediction that are truly reliable in order to avoid situations such as alarm fatigue.

Fig. 7 provides a visualization of the trade-off between F1-score and precision. Note that the calibrated probability with a threshold of 0.65 yields a slight drop in the F1-score while increasing precision by $7 \%$. This is a beneficial trade-off for our use case since that means that we can obtain more true positive detections without sacrificing the overall performance of the system. This degradation in F1-score was further explored in Fig. 8 by exploring the impact in recall. We observe that for an improvement of $7 \%$ in precision, the degradation in recall was of around $6 \%$.

Since the proposed criteria resets some of the original detections of the model, for completeness, Fig. 9 is intended to show the trade-off between uncertainty threshold values and the percentage of samples that are kept unchanged (i.e.: not set as negative prediction if outside of the threshold criterion). We notice that for a percentage of samples kept unchanged higher than $60 \%$, both criteria will provide similar AUC values. This plot motivates the previous analysis, in order to verify the impact of samples selection with other metrics such as precision and F1-score.

\section{DISCUSSION}

There are three main conclusions that we draw from the previous results:

BNNs improve performance beyond what is obtained by simply increasing model capacity. The results for WiderNet FCN 128 are averaged and summarized in Table VII The first experiments show that the Bayesian approach presented a modest and inconsistent improvement to Rad's approach, it improved the performance on Study 2 while it was slightly degraded for Study 1 (see Table IV) and summarized in Table VII Increasing model complexity for EDAQA dataset degraded performance for non-Bayesian models, making a case for possible overfitting. For WiderNet, the performance was enhanced in general when considering the Bayesian variants. One possible explanation is that applying the Bayesian framework on a model has a regularization effect [51], which for a model with lower capacity, such as Rad's model, result in lower performance. It is important to note that the AUC improvements for Bayesian approach were insensitive to further increases in capacity, leading us to believe that the framework has reached a limit on its performance. Additionally, it is interesting to note that for the EDAQA dataset, precision increases with larger Bayesian models whereas it decreases for larger non-Bayesian models. It is important to note that according to [52] a DL model approaches a Gaussian process as the number of layers of the DL model goes to infinity. Another important aspect to bring to discussion is that as shown by [46] a sufficient deep and wide model can even fit corrupted data since DL models have enough capacity to model very complex and even noisy data. However, based on the observations so far we have some evidence that the WiderNet model had benefited from the Bayesian approach, showing that not only deeper models benefit from such approach but also wider ones. It also shows that model capacity alone did not extract the "full potential" of the model. Additionally, the Bayesian approach gives us a relatively computationally cheap way of obtaining uncertainties from model predictions. One could argue that a simpler ensemble could also provide the same benefit, but based on our previous work [33] we have observed that random forests for example does not perform well 


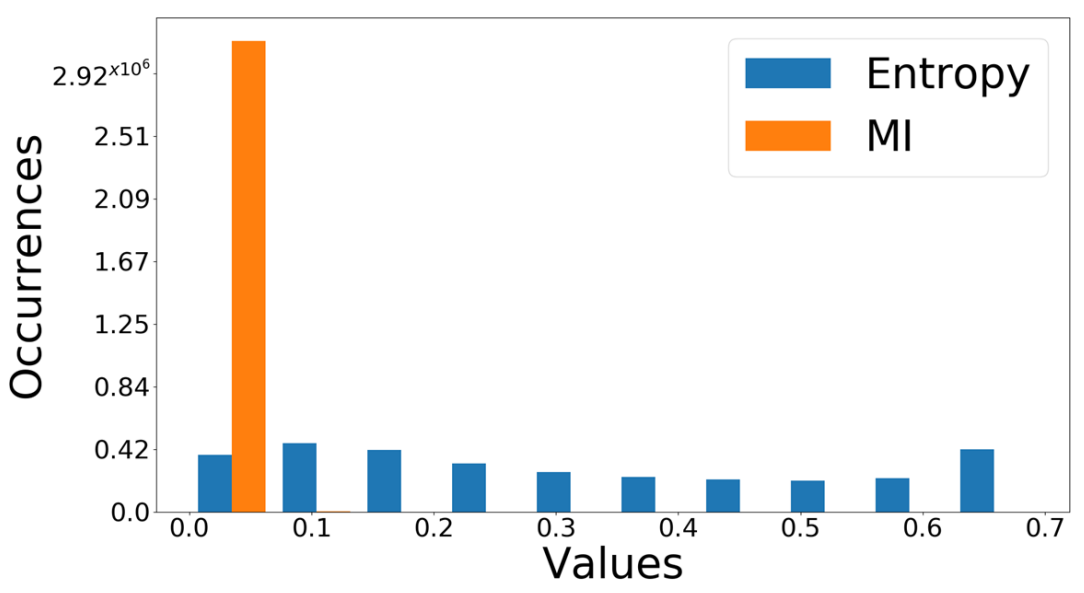

(a) WiderNet 8x, FCN 128 (correct)

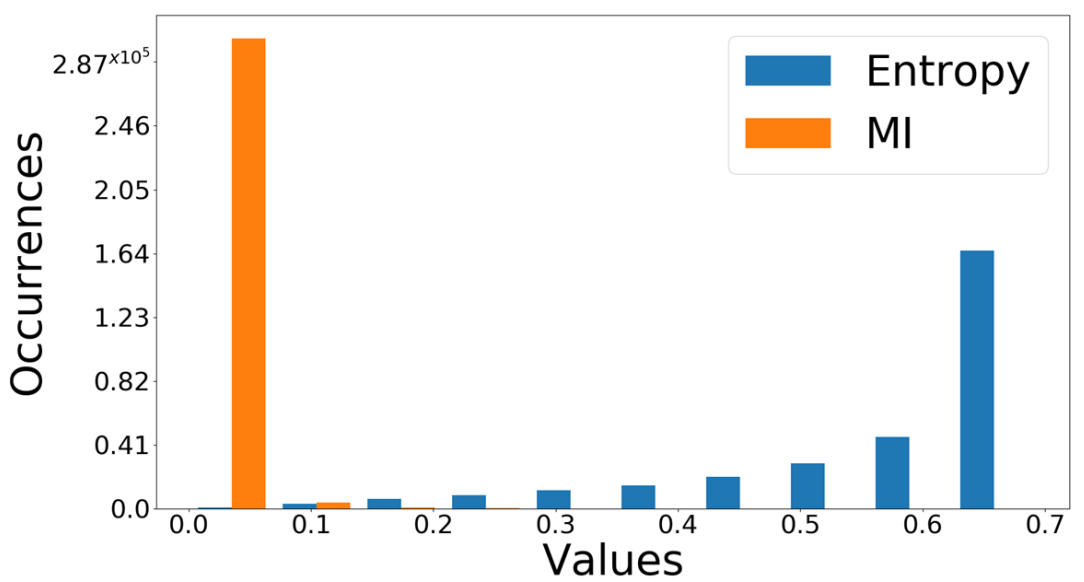

(b) WiderNet 8x, FCN 128 (incorrect)

Fig. 6: Distribution of correct and incorrect predictions for WiderNet 8x, FCN-128 on the ESDB dataset. Note that the entropy has different distributions for the correct vs. incorrect predictions. This indicate that it is a good feature for quantifying the uncertainty (i.e., higher entropy means higher likelihood of having an incorrect prediction). Best viewed in color.

for this dataset (and thus we used an SVM), therefore a DL approach was chosen for this work. While further investigation with computationally cheaper ensemble models is possible for uncertainty quantification, we leave that as future work.

Transfer learning reduces model variability. The evidence provided in section IV-B supports the claim for transfer learning which has an intuitive appeal: a model that has learned a similar domain will provide less variability when being retrained. An interesting unfolding of this result could be used to investigate the impact in model generalization, which we leave as future work.

The calibrated uncertainty can serve as a prediction quality indicator. Section $\mathrm{IV}-\mathrm{C}$ shows that calibrated probability provides slightly better improvements in precision for choosing good predictions. To further illustrate that, we have computed the ROC when removing the samples (and associated ground truth values) that do not meet the selection criterion and obtained an AUC of $98 \%$. Although this represents an unrealistic scenario, we have evidence that the remaining samples are in very close agreement with their respective ground truth values. Revealing that the uncertainty based thresholding really eliminates predictions with "poor quality". Fig. 10 illustrates this case by adding the new curve to former Fig. 4

\section{CONCLUSION}

In this work a comprehensive comparative study of methods to classify the body-rocking activity was presented. The methods were evaluated in light of a Bayesian approach. It was observed that a shallower model tends to not take advantage from the Bayesian approach. Additionally, the Bayesian approach was shown to provide superior performance benefits when applied to higher capacity models, as demonstrated with simple networks that were wider than the baseline model, which we called WiderNet. Although the experiments show this tendency, we acknowledge that more evaluations with other deeper and wider 


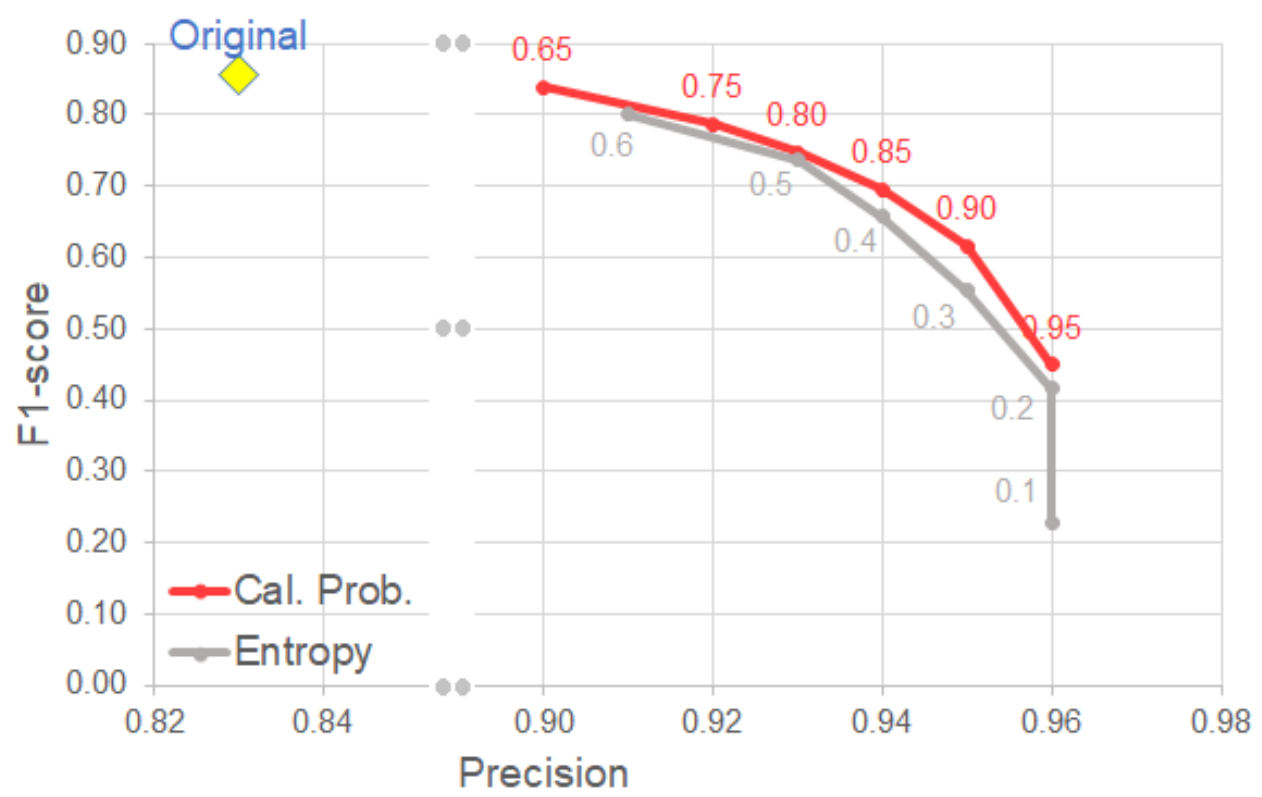

Fig. 7: F1-Score vs. Precision plot using uncertainty selection for ESDB dataset. The performances are obtained by setting the predictions of the model to a negative detection if their uncertainty follows below the specified calibrated probability or entropy thresholds. The original model is WiderNet 8x, FCN 128 with Bayesian approach and no selection. Note that the the curve produced by the cal. prob. is better than the entropy curve. Also, a threshold of 0.65 on the cal. prob. yields almost no drop in F1-score but and 7\% increase in precision. Best viewed in color.

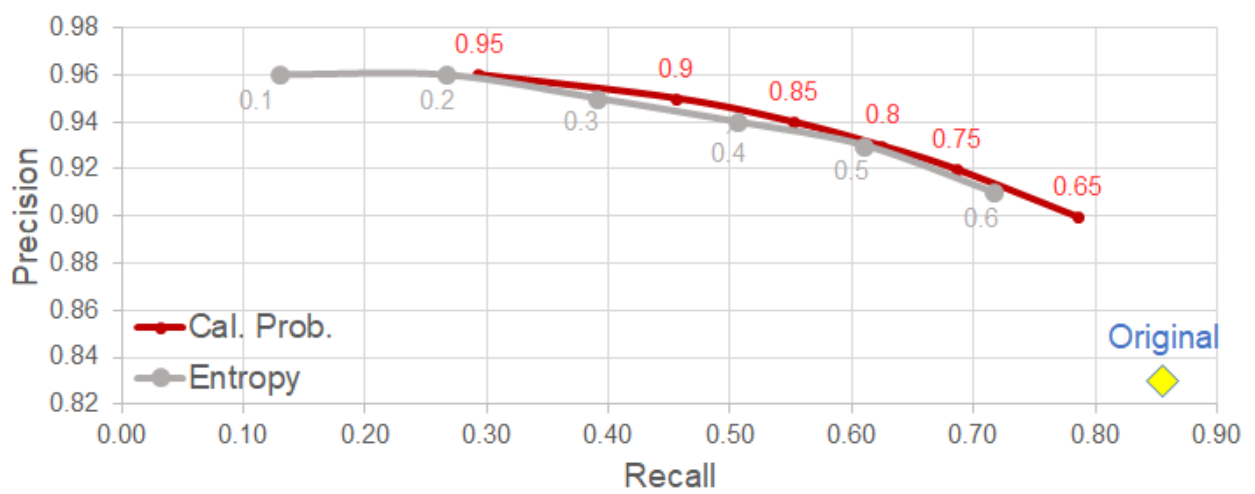

Fig. 8: Precision vs. Recall plot for uncertainty selection on ESDB dataset. The original model is WiderNet 8x, FCN 128 with Bayesian approach and no selection. Note that a threshold of 0.65 on the cal. prob. yields about an equal percentage of increase in precision and decrease in recall. Best viewed in color.

models, as well as other datasets, are needed in order to isolate the capacity effect v.s. Bayesian approach. Assuming precision as a better metric than F1-score for body-rocking classification, the calibrated probability and estimated entropy turned out to be useful criteria to establish a "reliable level" for model predictions, significantly improving model's precision, reducing the amount of false positives, and making the case to use such methods for real-time detection.

Bayesian DL is still a growing research area for which new insights are being shared. We foresee that the performance observed in this paper can be further improved by not only comprehensively evaluating deep architectures, but also exploring the effects of different priors for body-rocking classification and new ways of obtaining posteriors. The work of [53], for example, condensed a series of justifications for the use of cold posteriors on top of the fact that there is theoretical and experimental evidence that posterior predictive can be better than point estimators and that model averaging, in general, provide robust prediction. They show that cold posteriors improve the predictions of Stochastic Gradient-Monte Carlo Markov Chain based ensembles, which could also bring benefits for body-rocking classification. Applications such as body-rocking detection can largely benefit from the constant new outcomes from Bayesian DL. In order to make "live" body-rocking detection using BNNs viable, a clinical study evaluating user feedback for real-time detection and uncertainty threshold adjustment should be conducted, with devices equipped with real-time BNNs, that we leave as future work. 


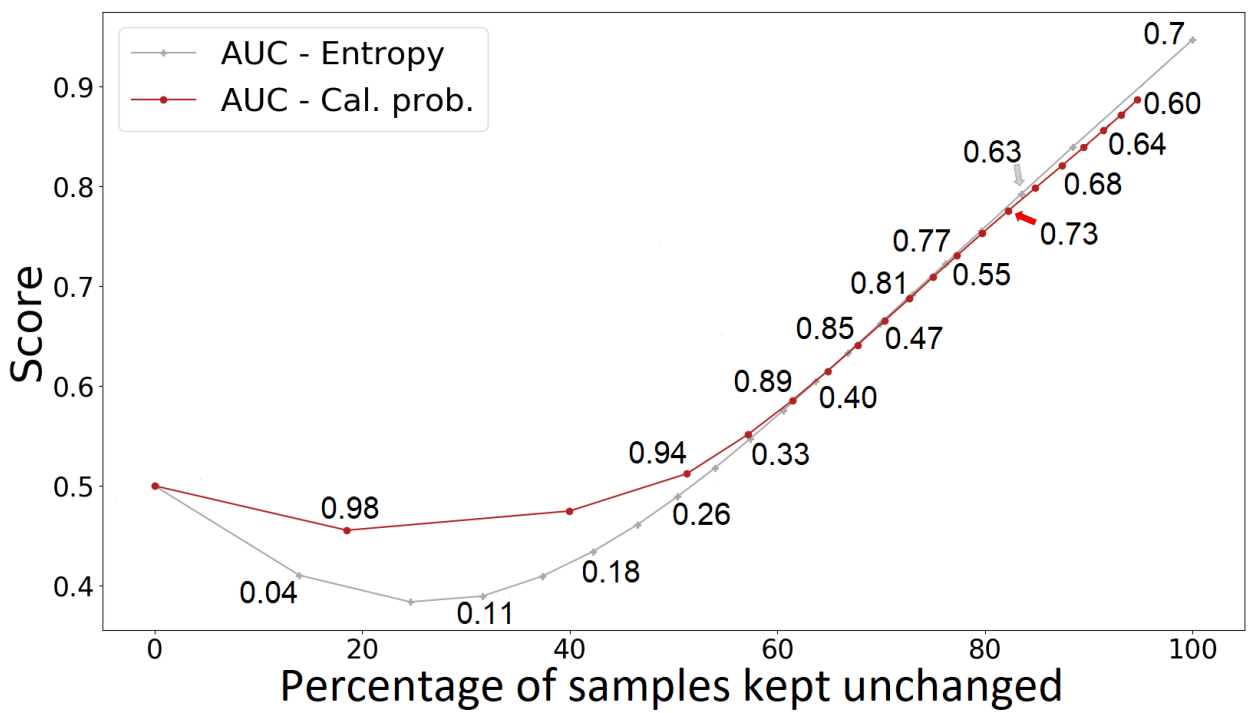

Fig. 9: AUC score vs. Percentage of samples greater kept unchanged for uncertainty selection on ESDB dataset. The original model is WiderNet 8x, FCN 128 with Bayesian approach. Best viewed in color.

TABLE VII: Summary of averaged performance for each dataset

\begin{tabular}{llrrr} 
& & Study 1 & Study 2 & ESDB \\
\hline Rad Original & AUC: & 0.896 & 0.906 & 0.916 \\
& F1: & 0.549 & 0.580 & 0.841 \\
& Precision & 0.620 & 0.680 & 0.810 \\
\hline Rad Bayes & AUC: & 0.891 & 0.920 & 0.925 \\
& F1: & 0.502 & 0.530 & 0.852 \\
& Precision & 0.690 & 0.720 & 0.820 \\
\hline WiderNet 2x & AUC: & 0.895 & 0.921 & 0.929 \\
& F1: & 0.584 & 0.636 & 0.855 \\
& Precision & 0.640 & 0.670 & 0.830 \\
\hline WiderNet 2x & AUC: & 0.941 & 0.958 & 0.943 \\
Bayes & F1: & 0.612 & 0.679 & 0.867 \\
& Precision & 0.660 & 0.710 & 0.860 \\
\hline WiderNet 4x & AUC: & 0.885 & 0.920 & 0.930 \\
& F1: & 0.587 & 0.653 & 0.857 \\
& Precision & 0.640 & 0.670 & 0.830 \\
\hline WiderNet 4x & AUC: & $\mathbf{0 . 9 4 8}$ & $\mathbf{0 . 9 6 1}$ & 0.946 \\
Bayes & F1: & 0.645 & 0.695 & $\mathbf{0 . 8 7 0}$ \\
& Precision & 0.680 & 0.710 & 0.860 \\
\hline WiderNet 8x & AUC: & 0.869 & 0.903 & 0.929 \\
& F1: & 0.554 & 0.602 & 0.856 \\
& Precision & 0.620 & 0.680 & 0.830 \\
\hline WiderNet 8x & AUC: & 0.945 & 0.960 & $\mathbf{0 . 9 4 7}$ \\
Bayes & F1: & $\mathbf{0 . 6 5 5}$ & $\mathbf{0 . 7 0 3}$ & $\mathbf{0 . 8 7 0}$ \\
& Precision & 0.690 & 0.720 & $\mathbf{0 . 8 7 0}$ \\
\hline WiderNet 16x & AUC: & 0.838 & 0.892 & 0.926 \\
& F1: & 0.529 & 0.570 & 0.852 \\
WiderNet 16x & AUrecision & 0.560 & 0.670 & 0.830 \\
\hline Fayes & F1: & 0.943 & 0.954 & 0.945 \\
& Precision & 0.654 & 0.700 & 0.866 \\
& & & $\mathbf{0 . 7 0 0}$ & $\mathbf{0 . 8 7 0}$
\end{tabular}




\section{REFERENCES}

[1] A. Czapliński, A. J. Steck, and P. Fuhr, "[tic syndrome].," Neurologia i neurochirurgia polska, vol. 36 3, pp. 493-504, 2002.

[2] H. S. Singer, "Stereotypic movement disorders.," Handbook of clinical neurology, vol. 100, pp. 631-9, 2011.

[3] E. M. Mahone, D. D. Bridges, C. Prahme, and H. S. Singer, "Repetitive arm and hand movements (complex motor stereotypies) in children.", The Journal of pediatrics, vol. 145 3, pp. 391-5, 2004.

[4] D. Muthugovindan and H. S. Singer, "Motor stereotypy disorders.," Current opinion in neurology, vol. 22 2, pp. 131-6, 2009.

[5] H. Troester, M. Brambring, and A. Beelmann, "The age dependence of stereotyped behaviours in blind infants and preschoolers.," Child: care, health and development, vol. 17 2, pp. 137-57, 1991.

[6] E. McHugh and J. L. Pyfer, "The development of rocking among children who are blind," Journal of Visual Impairment Blindness, vol. 93, pp. 82 95, 1999.

[7] N. Rafaeli-Mor, L. G. Foster, and G. Berkson, "Self-reported body-rocking and other habits in college students.," American journal of mental retardation : AJMR, vol. 104 1, pp. 1-10, 1999.

[8] J. M. Miller, H. S. Singer, D. D. Bridges, and H. R. Waranch, "Behavioral therapy for treatment of stereotypic movements in nonautistic children.," Journal of child neurology, vol. 21 2, pp. 119-25, 2006.

[9] A. Subki, M. Alsallum, M. N. Alnefaie, A. Alkahtani, S. Almagamsi, Z. Alshehri, R. Kinsara, and M. Jan, "Pediatric motor stereotypies: An updated review," Journal of pediatric neurology: JPN, vol. 15, 032017.

[10] R. L. da Silva, E. Stone, and E. Lobaton, "A feasibility study of a wearable real-time notification system for self-awareness of body-rocking behavior," 2019 41st Annual International Conference of the IEEE Engineering in Medicine and Biology Society (EMBC), pp. 3357-3359, 2019.

[11] X. Wang, Y. Gao, J. Lin, H. Rangwala, and R. Mittu, "A machine learning approach to false alarm detection for critical arrhythmia alarms," 2015 IEEE 14th International Conference on Machine Learning and Applications (ICMLA), pp. 202-207, 2015.

[12] L. M. Eerikäinen, J. Vanschoren, M. J. Rooijakkers, R. Vullings, and R. M. Aarts, "Reduction of false arrhythmia alarms using signal selection and machine learning.," Physiological measurement, vol. 37 8, pp. 1204-16, 2016.

[13] G. E. Hinton and R. M. Neal, "Bayesian learning for neural networks," 1995.

[14] D. J. C. MacKay, "A practical bayesian framework for backpropagation networks," Neural Computation, vol. 4, pp. 448-472, 1992.

[15] Y. Gal and Z. Ghahramani, "Dropout as a bayesian approximation: Representing model uncertainty in deep learning," ArXiv, vol. abs/1506.02142, 2016.

[16] B. Zhong, R. L. D. Silva, M. Li, H. Huang, and E. Lobaton, "Environmental context prediction for lower limb prostheses with uncertainty quantification," IEEE Transactions on Automation Science and Engineering, pp. 1-13, 2020.

[17] B. Zhong, H. Huang, and E. Lobaton, "Reliable vision-based grasping target recognition for upper limb prostheses." IEEE transactions on cybernetics, vol. PP, 2020.

[18] S. Thakur, H. van Hoof, J. C. G. Higuera, D. Precup, and D. Meger, "Uncertainty aware learning from demonstrations in multiple contexts using bayesian neural networks," in 2019 International Conference on Robotics and Automation (ICRA), pp. 768-774, 2019.

[19] A. Akbari and R. Jafari, "Personalizing activity recognition models through quantifying different types of uncertainty using wearable sensors," IEEE Transactions on Biomedical Engineering, vol. 67, no. 9, pp. 2530-2541, 2020.

[20] K. H. Gilchrist, M. Hegarty-Craver, R. P. K. B. Christian, S. Grego, A. C. Kies, and A. C. Wheeler, "Automated detection of repetitive motor behaviors as an outcome measurement in intellectual and developmental disabilities.," Journal of autism and developmental disorders, vol. 48 5, pp. 1458-1466, 2018.

[21] U. Grossekathöfer, N. V. Manyakov, V. Mihajlovic, G. Pandina, A. Skalkin, S. Ness, A. Bangerter, and M. S. Goodwin, "Automated detection of stereotypical motor movements in autism spectrum disorder using recurrence quantification analysis," in Front. Neuroinform., 2017.

[22] C.-H. Min, A. H. Tewfik, Y. Kim, and R. Menard, "Optimal sensor location for body sensor network to detect self-stimulatory behaviors of children with autism spectrum disorder," 2009 Annual International Conference of the IEEE Engineering in Medicine and Biology Society, pp. 3489-3492, 2009.

[23] M. S. Goodwin, M. Haghighi, Q. Tang, M. Akçakaya, D. Erdogmus, and S. S. Intille, "Moving towards a real-time system for automatically recognizing stereotypical motor movements in individuals on the autism spectrum using wireless accelerometry," in UbiComp, 2014.

[24] M. S. Goodwin, S. S. Intille, F. Albinali, and W. F. Velicer, "Automated detection of stereotypical motor movements.," Journal of autism and developmental disorders, vol. 41 6, pp. 770-82, 2011.

[25] C.-H. Min and A. H. Tewfik, "Automatic characterization and detection of behavioral patterns using linear predictive coding of accelerometer sensor data," 2010 Annual International Conference of the IEEE Engineering in Medicine and Biology, pp. 220-223, 2010.

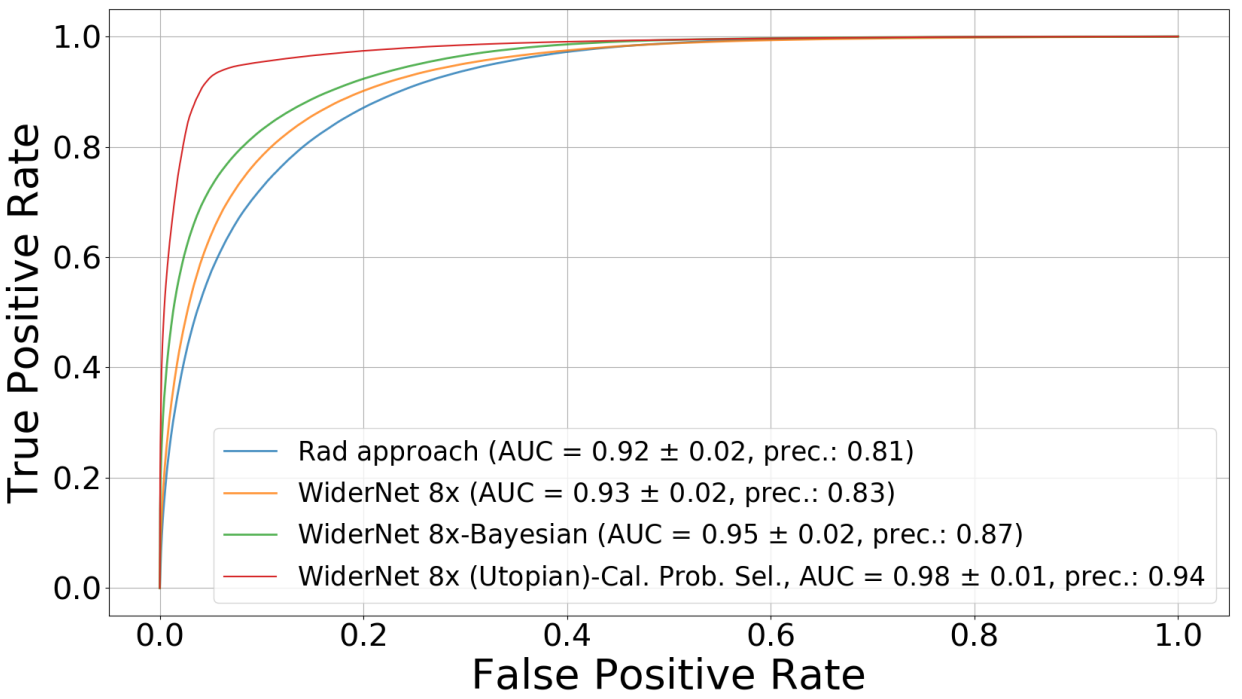

Fig. 10: Comparison for ESDB dataset considering a Utopian case where ground truth values are also removed if the associated calibrated probability is smaller than the selection threshold. Best viewed in color. 
[26] F. Albinali, M. S. Goodwin, and S. S. Intille, "Recognizing stereotypical motor movements in the laboratory and classroom: a case study with children on the autism spectrum," in UbiComp, 2009.

[27] N. M. Rad, S. M. Kia, C. Zarbo, T. van Laarhoven, G. Jurman, P. Venuti, E. Marchiori, and C. Furlanello, "Deep learning for automatic stereotypical motor movement detection using wearable sensors in autism spectrum disorders," Signal Processing, vol. 144, pp. 180-191, 2018.

[28] L. Sadouk, T. Gadi, and E. H. Essoufi, "A novel deep learning approach for recognizing stereotypical motor movements within and across subjects on the autism spectrum disorder," in Comp. Int. and Neurosc., 2018.

[29] N. M. Rad and C. Furlanello, "Applying deep learning to stereotypical motor movement detection in autism spectrum disorders," 2016 IEEE 16th International Conference on Data Mining Workshops (ICDMW), pp. 1235-1242, 2016.

[30] N. M. Rad, S. M. Kia, C. Zarbo, G. Jurman, P. Venuti, and C. Furlanello, "Stereotypical motor movement detection in dynamic feature space," 2016 IEEE 16th International Conference on Data Mining Workshops (ICDMW), pp. 487-494, 2016.

[31] S. J. Pan and Q. Yang, "A survey on transfer learning," IEEE Transactions on Knowledge and Data Engineering, vol. 22, pp. 1345-1359, 2010.

[32] H. chen Wu, E. D. Lemaire, and N. C. Baddour, "Combining low sampling frequency smartphone sensors and video for a wearable mobility monitoring system," F1000Research, vol. 3, 2015.

[33] R. L. da Silva, E. Stone, and E. Lobaton, "A feasibility study of a wearable real-time notification system for self-awareness of body-rocking behavior," 201941 st Annual International Conference of the IEEE Engineering in Medicine and Biology Society (EMBC), pp. 3357-3359, 2019.

[34] R. L. da Silva, "Education Services for the Deaf and Blind of the North Carolina Department of Public Instruction (ESDB dataset)," Oct. 2021.

[35] N. Srivastava, G. E. Hinton, A. Krizhevsky, I. Sutskever, and R. Salakhutdinov, "Dropout: a simple way to prevent neural networks from overfitting," $J$. Mach. Learn. Res., vol. 15, pp. 1929-1958, 2014.

[36] C. K. I. Williams, "Computing with infinite networks," in NIPS, 1996

[37] C. M. Bishop, "Pattern recognition and machine learning," (United States of America), p. 463, Springer, 2006.

[38] B. Zhong, "Reliable deep learning for intelligent wearable systems.," 2020.

[39] Y. Gal and Z. Ghahramani, "Dropout as a bayesian approximation: Appendix," arXiv: Machine Learning, 2015.

[40] Y. Gal and Z. Ghahramani, "Dropout as a bayesian approximation: Representing model uncertainty in deep learning," in international conference on machine learning, pp. 1050-1059, 2016.

[41] L. Smith and Y. Gal, "Understanding measures of uncertainty for adversarial example detection," arXiv preprint arXiv:1803.08533, 2018.

[42] A. Kendall and Y. Gal, "What uncertainties do we need in bayesian deep learning for computer vision?," in Advances in neural information processing systems, pp. 5574-5584, 2017.

[43] C. E. Shannon, "A mathematical theory of communication," Bell system technical journal, vol. 27, no. 3, pp. 379-423, 1948.

[44] N. Houlsby, F. Huszár, Z. Ghahramani, and M. Lengyel, "Bayesian active learning for classification and preference learning," arXiv preprint arXiv:1112.5745, 2011.

[45] B. Zhong, R. L. D. Silva, M. Li, H. Huang, and E. Lobaton, "Environmental context prediction for lower limb prostheses with uncertainty quantification," IEEE Transactions on Automation Science and Engineering, pp. 1-13, 2020.

[46] C. Zhang, S. Bengio, M. Hardt, B. Recht, and O. Vinyals, "Understanding deep learning requires rethinking generalization," ArXiv, vol. abs/1611.03530, 2017.

[47] M. Vladimirova, J. Verbeek, P. Mesejo, and J. Arbel, "Understanding priors in bayesian neural networks at the unit level," in ICML, 2019.

[48] Á. Tejero-Cantero, J. Boelts, M. Deistler, J.-M. Lueckmann, C. Durkan, P. J. Gonccalves, D. S. Greenberg, J. H. M. C. Neuroengineering, D. Electrical, C. Engineering, T. U. Munich, S. of Informatics, U. Edinburgh, N. S. Analysis, C. of Advanced European Studies, Research, Bonn, M.-D. M. Learning, C. for Materials, C. Research, H.-Z. Geesthacht, M. L. in Science, U. Tubingen, E. Inference, M. P. I. for Intelligent Systems, and Tubingen, "Sbi - a toolkit for simulation-based inference," J. Open Source Softw., vol. 5, p. 2505, 2020.

[49] D. Silvestro and T. Andermann, "Prior choice affects ability of bayesian neural networks to identify unknowns," ArXiv, vol. abs/2005.04987, 2020.

[50] B. Zhong, R. L. da Silva, M. Tran, H. Huang, and E. Lobaton, "Efficient environmental context prediction for lower limb prostheses," IEEE Transactions on Systems, Man, and Cybernetics: Systems, pp. 1-15, 2021.

[51] A. Kendall, V. Badrinarayanan, and R. Cipolla, "Bayesian segnet: Model uncertainty in deep convolutional encoder-decoder architectures for scene understanding," ArXiv, vol. abs/1511.02680, 2017.

[52] Y. Gal and Z. Ghahramani, "Bayesian convolutional neural networks with bernoulli approximate variational inference," arXiv preprint arXiv:1506.02158, 2015.

[53] F. Wenzel, K. Roth, B. S. Veeling, J. Swiatkowski, L. Tran, S. Mandt, J. Snoek, T. Salimans, R. Jenatton, and S. Nowozin, "How good is the bayes posterior in deep neural networks really?," in ICML, 2020.

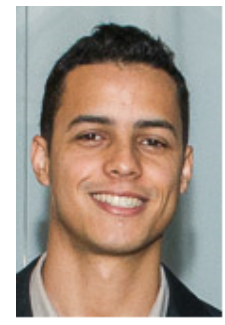

Rafael Luiz da Silva received the undergraduate degree in technology of industrial automation from Instituto Federal de Educaço, Ciência e Tecnologia de São Paulo, Brazil, in 2008 and the M.Sc. degree in electrical engineering from Escola Politécnica of University of São Paulo, Brazil, in 2013. He is currently pursuing the Ph.D. degree in electrical engineering at NC State University, Raleigh, NC, USA. From 2008 to 2016 he was a development analyst with Continental Automotive Group, Guarulhos, SP, Brazil, working on automotive embedded systems. His research interests includes computer vision, machine learning and signal processing. 


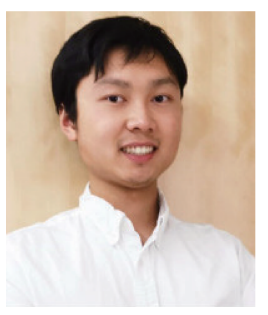

Boxuan Zhong received the B.E. degree in electronics and information engineering from the Huazhong University of Science and Technology, Wuhan, China, in 2015. He is currently pursuing the Ph.D. degree in electrical and computer engineering with North Carolina State University, Raleigh, NC, USA.

His current research interests include computer vision, machine learning, and robotics.

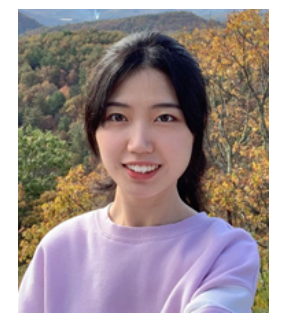

Yuhan Chen is a Ph.D. student advised by Dr. Lobaton in the Active Robotic Sensing (ARoS) Lab at North Carolina State University. She received the B.S. degree in Electrical and Communication Engineering from the South China Normal University. Her current research interests include machine learning, bio-signal processing, and computer vision.

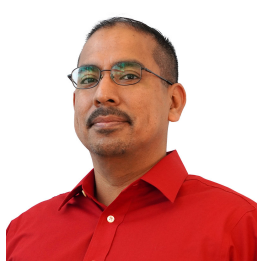

Edgar Lobaton received the Ph.D. degree in electrical engineering and computer sciences from the University of California, Berkeley, CA, USA, in 2009. He currently an Associate Professor in the Department of Electrical and Computer Engineering, North Carolina State University, Raleigh, NC, USA. He was engaged in research at Alcatel-Lucent Bell Labs in 2005 and 2009.

Dr. Lobaton was awarded the 2009 Computer Innovation Fellows Postdoctoral fellowship and conducted research in the Department of Computer Science, University of North Carolina at Chapel Hill, Chapel Hill, NC, USA, from 2009 to 2011. His research interests include pattern recognition, computer vision, and robotics. 